\title{
Proton-neutron multiplet states and isomers in the odd-odd nucleus ${ }^{122}$ I
}

\author{
B. Moon $\odot,{ }^{1,2}$ C.-B. Moon, ${ }^{3,}{ }^{*}$ G. D. Dracoulis, ${ }^{4}$ R. A. Bark,${ }^{4,5}$ A. P. Byrne,${ }^{4}$ P. A. Davidson, ${ }^{4}$ \\ G. J. Lane, ${ }^{4}$ T. Kibédi, ${ }^{4}$ A. N. Wilson, ${ }^{4}$ and C. Yuan ${ }^{6}$ \\ ${ }^{1}$ Department of Physics, Korea University, Seoul 02841, Republic of Korea \\ ${ }^{2}$ Center for Extreme Nuclear Matters, Korea University, Seoul 02841, Republic of Korea \\ ${ }^{3}$ Faculty of Science, Hoseo University, Chung-Nam 31499, Republic of Korea \\ ${ }^{4}$ Department of Nuclear Physics, Research School of Physical Sciences and Engineering, \\ Australian National University, Canberra ACT2601, Australia \\ ${ }^{5}$ iThemba Laboratories, P.O. Box 722, Somerset West 7129, South Africa \\ ${ }^{6}$ Sino-French Institute of Nuclear Engineering and Technology, Sun Yat-Sen University, Zhuhai 519-082, China
}

(Received 4 April 2019; published 13 August 2019)

\begin{abstract}
The level structure of the odd-odd iodine nucleus ${ }^{122} \mathrm{I}$ has been studied by the ${ }^{120} \mathrm{Sn}\left({ }^{7} \mathrm{Li}, 5 n\right){ }^{122} \mathrm{I}$ reaction at $E_{\mathrm{lab}}=58 \mathrm{MeV}$. Two chopped beams from the 14UD (Unit Doubled) tandem heavy-ion accelerator at the Australian National University were used; one was a nanosecond (ns) pulsed-beam with separation of 1.7 microseconds $(\mu \mathrm{s})$ and the second one was a $60-\mu \mathrm{s}$ beam separated by $880 \mu \mathrm{s}$. Through in-beam and out-of-beam $\gamma$-ray spectroscopy, the complex proton-neutron multiplet states, including isomers with half-lives of a few tens of ns to a few tens of $\mu \mathrm{s}$, have been identified. Collective states based on the proton $h_{11 / 2}$ orbital exhibit two kinds of structure depending upon the coupled neutron orbital: a quadrupole vibration when involving the $h_{11 / 2}$ orbital and a $\gamma$-soft deformed rotation when involving the $g_{7 / 2}$ orbital. With spherical shell model and deformed shell-model calculations, the observed level structures are explained.
\end{abstract}

DOI: 10.1103/PhysRevC.100.024319

\section{INTRODUCTION}

With proton number $Z=53$, iodine (I) lies between the spherical nuclei associated with the closed proton shell at $Z=$ 50 and deformed nuclei such as $\mathrm{Xe}$ and $\mathrm{Ba}$. Consequently, I isotopes are expected to exhibit a variety of structural changes over spherical and collective phases, according to specific underlying quasiparticle configurations. In the oddmass isotopes, the low-lying states arise mainly from proton excitations in the $g_{7 / 2}, d_{5 / 2}$, and $h_{11 / 2}$ orbitals. In addition, the holelike $g_{9 / 2}$ orbital below the $Z=50$ shell gap contributes to the occurrence of a collective structure. In particular, the highangular momentum intruder $h_{11 / 2}$ orbital plays a distinctive role in shaping nuclear structure in this region. Indeed, the collective bands built on the proton $h_{11 / 2}$ orbital in odd-mass I, $62 \leqslant N \leqslant 74$ [1-8], have been observed to dominate the yrast sequence. For odd-mass $\mathrm{Te}$ and $\mathrm{Xe}$, dominant yrast states are also built on the neutron $h_{11 / 2}$ orbital. Thus, it is expected that the positive-parity states based on the proton $h_{11 / 2}$ orbital coupled to the neutron $h_{11 / 2}$ orbital should appear dominantly in odd-odd I isotopes. Because of a narrow energy space and the occurrence of isomeric states in between the proton-neutron multiplet states, the study of odd-odd nuclei is challenging for making excitation energy and spin-parity assignments.

In this work for ${ }^{122} \mathrm{I}$, we present a detailed level construction including several isomers in odd-odd ${ }^{122} \mathrm{I}$ that includes

*cbmoon@hoseo.edu the absolute excitation energies. Using a large-scale spherical shell model and deformed shell-model calculations, we discuss the emerging features from proton-neutron configurations such as isomeric states with half-lives longer than a few tens of ns, collective states associated with vibration and rotation, and noncollective oblate states due to multinucleon alignments. Some partial results from the current experiment were reported earlier [9-11]. After our reports, a similar experimental result for this nucleus was presented by another group $[12,13]$.

\section{EXPERIMENTAL PROCEDURES}

The excited states of ${ }^{122} \mathrm{I}$ were produced through the ${ }^{120} \mathrm{Sn}$ $\left({ }^{7} \mathrm{Li}, 5 n\right)$ reaction at a beam energy of $58 \mathrm{MeV}$. Experiments were performed in two steps, initially with a 1-ns pulsed beam separated by $1.7 \mu \mathrm{s}$, and then with a $60-\mu \mathrm{s}$ chopped beam separated by $880 \mu \mathrm{s}$. The target consisted of a selfsupporting foil of ${ }^{120} \mathrm{Sn}$, enriched to $92.2 \%$, with a thickness of $3 \mathrm{mg} / \mathrm{cm}^{2} . \gamma$ rays emitted during the decay of the excited states were detected using the CAESAR array, which consisted of 6 Compton-suppressed high-purity germanium (HPGe) detectors and two low-energy photon (LEP) detectors. $\gamma$ rays were collected both during the period that the beam was incident on the target and the "out-of-beam" period, allowing the detection of decays from isomeric states. Two sets of data were collected: (i) a singles measurement was performed in which all $\gamma$ rays were timed relative to the beam pulse and (ii) a $\gamma-\gamma$ coincidence measurement was performed, again with timing relative to the beam pulse. The time difference 
between any two $\gamma$ rays in one event could then be reconstructed in subsequent analysis from the two times relative to the beam pulse.

For identifying isomers in the microsecond region, beam- $\gamma$ measurements were done using a chopped beam of $60-\mu \mathrm{s}$ pulses, separated by $880 \mu$ s. The HPGe detectors recorded off-beam $\gamma$-ray singles spectra in two-parameter $E_{\gamma}$ versus time mode as well as $\gamma-\gamma$ prompt coincidence events. A fast veto was used to remove $\gamma$ rays occurring in the $60-\mu \mathrm{s}$ inbeam period to increase the sensitivity to the $\gamma$ rays following an isomer. The data collected to disk included energy, time, and detector identification words. Efficiency and energy calibrations were performed with ${ }^{152} \mathrm{Eu}$ and ${ }^{133} \mathrm{Ba} \gamma$-ray sources, the latter to provide good calibration information at low energies. For off-line data analysis, the following procedures were done.

\section{A. $\gamma-\gamma$ coincidence}

The data were sorted, following energy gain and time matching, into $4096 \times 4096$ channel matrices with the following constraints on the $\gamma-\gamma$ coincidence relationships. The first matrix requires two $\gamma$ rays occur within \pm 40 ns of each other. On the other hand, the second matrix requires a less stringent condition where the time relationship was relaxed to $\pm 140 \mathrm{~ns}$ in order to include low-energy $\gamma$ rays which suffer from time walk in the detectors, where the $\gamma$ events are required to occur between beam bursts with the additional condition that they are separated in time between \pm 50 ns and \pm 850 ns. This matrix then allows for two event-projection types. One of these types is the projection of those events that precedes an isomer by gating on transitions that follow it, called early delayed. The other type is the projection of those events that follow an isomer by gating on transitions that feed it, called late delayed.

\section{B. $\gamma-\gamma$ time}

In order to isolate the lifetimes of individual states, the coincidence data were also sorted into three-dimensional $\gamma_{\text {start }}-\gamma_{\text {stop }}$-time matrices. The time relationship between pairs of $\gamma$ rays could be obtained from such matrices by taking background-subtracted $\gamma$-ray gates on transitions preceding and following any state. As an example, we provide the projected spectra for energies and timings correlated with the 159-keV transition in Fig. 1. In contrast, Fig. 2 describes two timing curves, one from $\gamma_{1}$ (start) $-\gamma_{2}$ (stop) and another from the reverse transition, which, as expected, are symmetrical in shape.

\section{C. $\gamma$ time}

Two-dimensional matrices were generated of $\gamma$-ray energy versus their time of arrival with respect to the beam pulse. Matrices were constructed corresponding to the different beam on-off periods: (a) $1 \mathrm{~ns}$ on, $1712 \mathrm{~ns}$ off and (b) $60 \mu \mathrm{s}$ on, $880 \mu$ s off, facilitating the measurement of the short-lived and the long-lived states. The singles data were put into matrices with $\gamma$-ray energies on one axis and time on the other, with two matrices constructed, one containing data from the HPGe
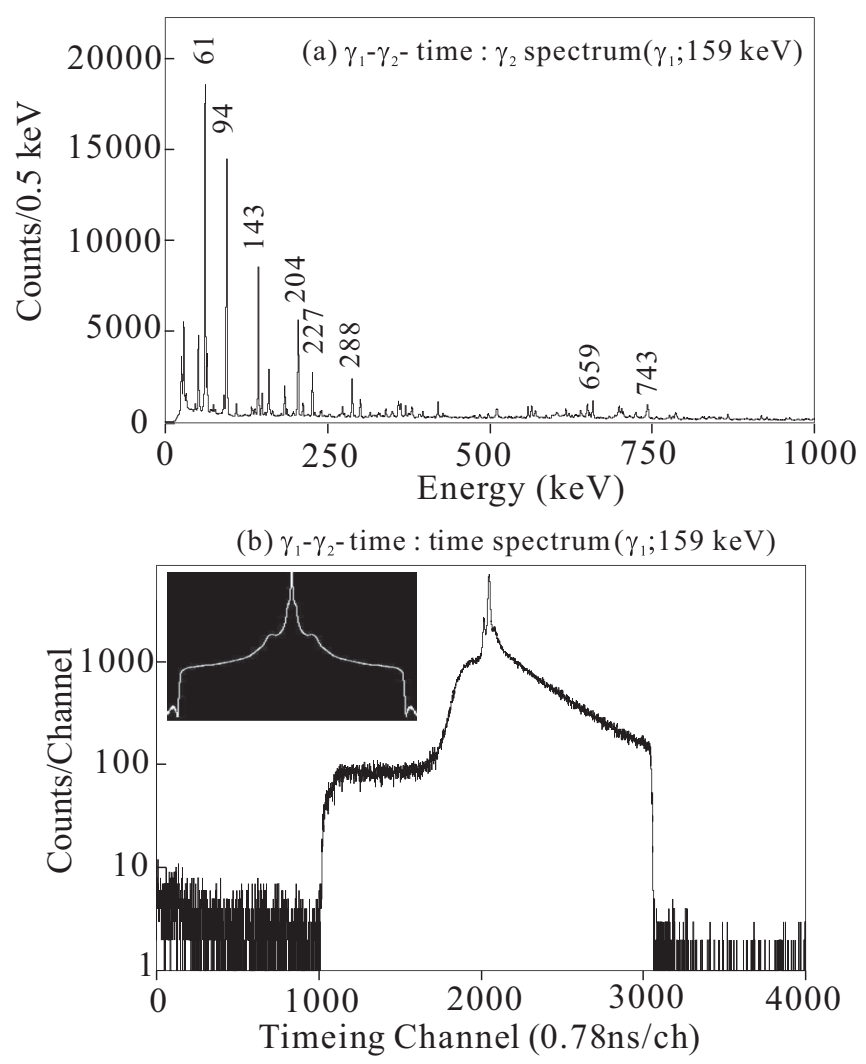

FIG. 1. (a) $\gamma$ rays $\left(\gamma_{2}\right)$ associated with the coincidence of the 159-keV $\left(\gamma_{1}\right)$ transition in $\gamma_{1}-\gamma_{2}$-time events. (b) Time-difference spectrum for the $\gamma_{1}(159-\mathrm{keV})-\gamma_{2}$ time. The right region indicates the decay curve for the $159-\mathrm{keV}$ transition. The inset in panel (b) shows the total time-difference spectrum. The shoulders in the time-difference spectra are due to time walk experienced by lowenergy $\gamma$ rays and $\mathrm{x}$ rays.

detectors and another containing data from the LEP detectors. Figure 3 displays delayed singles $\gamma$-ray peaks detected by the LEP detectors during these two beam- $\gamma$ measurements. Because of the high-energy resolution of the LEP detector, one can see that the $65-\mathrm{keV}$ transitions were found to be a

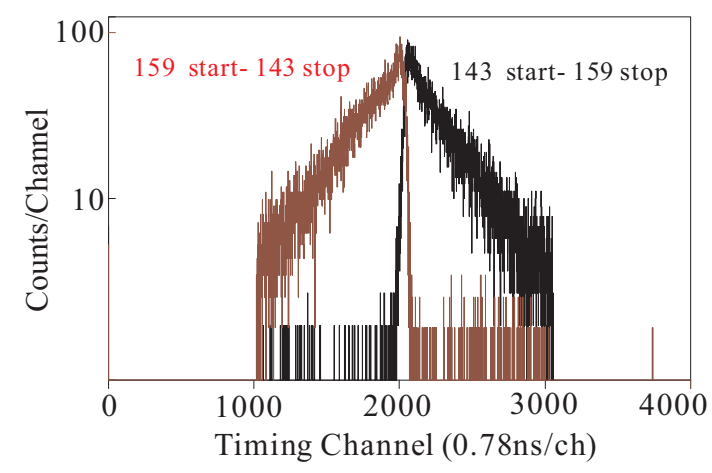

FIG. 2. $\gamma-\gamma$-time spectra gated on the 143- and 159-keV transitions. The right curve indicates that the isomeric state is below the start transition. The left curve indicates that the isomeric state is above the start transition. 

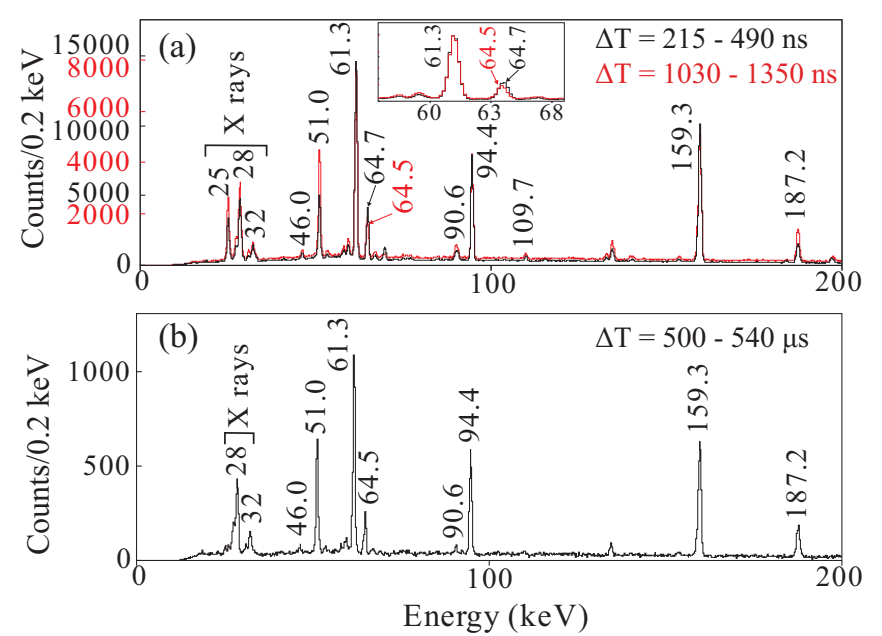

FIG. 3. Delayed singles $\gamma$-ray spectra from the ${ }^{120} \mathrm{Sn}\left({ }^{7} \mathrm{Li}, 5 n\right)$ reaction at $58 \mathrm{MeV}$ under varied beam off conditions, $\Delta T$. (a) For the beam on-beam off, $1 \mathrm{~ns}-1.7 \mu \mathrm{s}$, measurements; $\Delta T=215-490 \mathrm{~ns}$ is the black line and $\Delta T=1030-1350 \mathrm{~ns}$ is the dotted red (gray) line. (b) For the beam on-beam off, $60-880 \mu \mathrm{s}$, measurements; $\Delta T=$ 500-540 $\mu$ s. Notice the difference of energies between the 64.5and $64.7-\mathrm{keV}$ peaks and the difference of relative intensities in the 51-keV transitions. In panel (a), the inset represents the separated spectra of the 64.5 - and $64.7-\mathrm{keV}$ double peaks where the $64.5-\mathrm{keV}$ transition indicates a decaying transition from a longer isomer than an associated isomer with the 64.7-keV transition.

doublet with a $0.2-\mathrm{keV}$ difference. This identification plays a decisive role in determining the absolute excitation energies.

The coincidence matrices were constructed by having either all combinations of HPGe detectors on both axes or any of the six HPGe detectors on one axis and either of the LEP detectors on the other. Multipolarity information can be extracted from the data using the method of directional correlation of oriented states (DCO ratios). The coincidence events were sorted into an asymmetric matrix with $\gamma$-ray energies detected in the two detectors at $97^{\circ}$ along one axis and $\gamma$-ray energies detected in either the two detectors at $48^{\circ}$ or the two detectors at $145^{\circ}$ along the other axis. The DCO ratios of the transitions, $R=I\left(48^{\circ}+145^{\circ}\right) / I\left(97^{\circ}\right)$, were then determined wherever possible. In the present, the ratios were determined by gating on stretched quadrupole transitions in the band sequence preceding or following the transition of interest. When both the gating and the observed transitions are stretched transitions, the DCO values are $R \approx 1.0$; if the gate is set on a stretched quadrupole (dipole) transition and one looks at a stretched dipole (quadrupole) transition, the values are $R \approx 0.6$ (1.5). According to the total DCO data analysis, the observed $M 1 / E 2$ transitions in collective bands were found to have a stretched dipole-like character. In the present work, the $R$ values were mainly deduced from the gates on the stretched quadrupole transitions. It is worthwhile to know that, however, the DCO ratios are meaningful only when the orientation of a compound nucleus is properly preserved. Hence, the long half-lives of the isomers preclude any DCO measurements since angular momentum alignments are washed out during such long decaying times. Therefore, we applied the intensity balance to extracting a conversion electron coefficient for making multipolarity assignments to the transitions depopulating the isomers. We summarize experimental results associated with the $\gamma$-ray transitions belonging to ${ }^{122}$ I in Table I, where the energies, relative intensities, DCO ratios, and spin-parity assignments are presented.

\section{EXPERIMENTAL RESULTS AND LEVEL SCHEME}

Figure 4 illustrates the level scheme of ${ }^{122} \mathrm{I}$ as obtained from the present work. As shown in this scheme, we have delineated the complex low-spin states and established several collective bands. Furthermore, we have identified several isomeric states with half-lives ranging from a few tens of nanoseconds to tens of microseconds. It should be noted that the present level scheme disagrees with that reported earlier [14] where the dominant states, which we have assigned as positive parity, band 1 in Fig. 4, were assigned to be negative parity based on a $9^{-}$band head. Our result was confirmed by a later work $[12,13]$. Furthermore, the low-lying structures on which collective states are based were not defined until our work. Low-lying states in ${ }^{122} \mathrm{I}$ had been studied from the radioactive $\beta^{+}$decay of ${ }^{122} \mathrm{Xe}$ and the spin parity of the ground state had been assigned as $J^{\pi}=1^{+}$with a half-life of 3.63(6) $\mathrm{m}$ [15]. Wu et al. [16] found an isomeric state of $7^{-}$with a half-life of $80(8) \mu$ s through delayed $\gamma$-ray spectroscopy using a recoil mass separator. Despite its excitation energy being unknown, two separate sequences of the 274-(352)-(376)-(406) $\mathrm{keV}$ and the 185-301-340-360-381$397 \mathrm{keV}$ transitions were found to feed the isomer. One can see that on one side, the 274-keV-based sequence corresponds to band 7 and, on the other side, the 185-, 301-, 340-, 360-, $381-$, and $397-\mathrm{keV}$ peaks are members of band 1, from the $16^{+}$state at $3008 \mathrm{keV}$ to the $8^{+}$state at $390 \mathrm{keV}$.

As shown in Fig. 3, numerous delayed transitions support the existence of long-lived isomeric states in ${ }^{122} \mathrm{I}$. In a singles mode as well as in the prompt $\gamma-\gamma$ coincidence data, 61-, 94-, and $159-\mathrm{keV}$ transitions are most strongly populated. In the experiment with 1-ns pulsed beams with a separation of $1.7 \mu \mathrm{s}$, the three $61-94-159-\mathrm{keV}$ transitions were found to be fed from an isomer as they are correlated with each other. This indicates that the level at $315 \mathrm{keV}$ is likely an isomer. Figure 5 shows prompt $\gamma-\gamma$ coincidence spectra gated by the 61-, 94-, and 159-keV transitions in which preceding transitions above the isomer at $315 \mathrm{keV}$ are too sparse in population to be observed. On the other hand, Fig. 6(a) represents the $\gamma$ rays feeding the isomer in an early delayed coincidence gated by the $159-\mathrm{keV}$ transition that depopulates it and Fig. 6(b) represents the $\gamma$ rays depopulating the isomer in late delayed coincidence gated by the $143-\mathrm{keV}$ transition that feeds it. These early- and late-delayed coincidence spectra indicate that the $315-\mathrm{keV}$ state is likely a long-lived isomer with a half-life above a few tens of ns. For the assignment of multipolarities to these 61-, 95-, and $159-\mathrm{keV}$ transitions, we apply the intensity balance argument. According to the result from a previous work [14], the 61-keV transition has been reported as $M 1$. Given this $M 1$ of the $61-\mathrm{keV}$ transition, the extracted electron conversion coefficients for the 94- and $159-\mathrm{keV}$ transitions are 3.26 and 1.49 , respectively. These 
TABLE I. $\gamma$-ray energies, relative intensities, DCO values, multipolarities of the transitions belonging to ${ }^{122}$ I, together with spin assignments of the initial and final levels. Isomeric states include their half-lives.

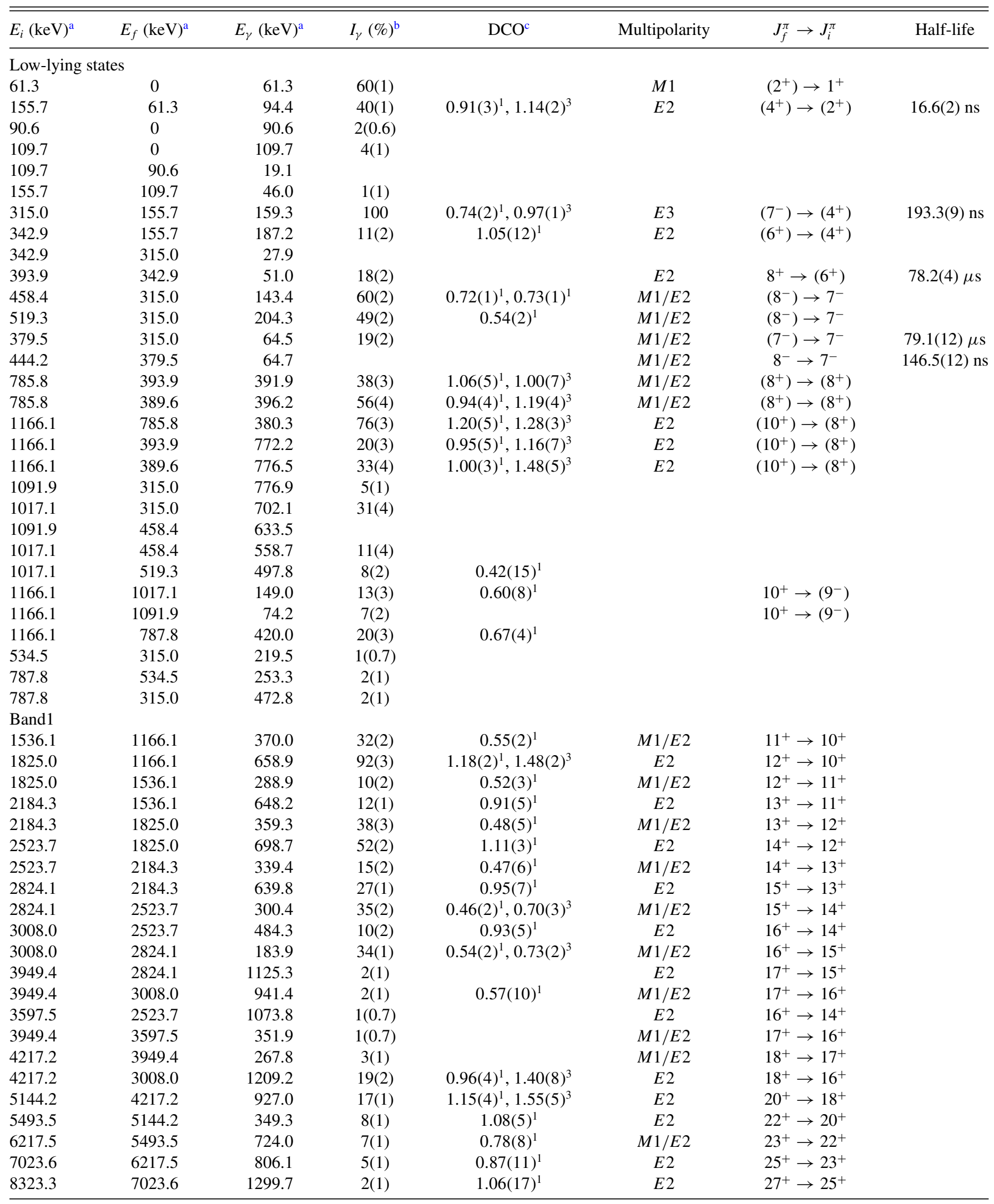


TABLE I. (Continued.)

\begin{tabular}{|c|c|c|c|c|c|c|}
\hline$E_{i}(\mathrm{keV})^{\mathrm{a}}$ & $E_{f}(\mathrm{keV})^{\mathrm{a}}$ & $E_{\gamma}(\mathrm{keV})^{\mathrm{a}}$ & $I_{\gamma}(\%)^{\mathrm{b}}$ & $\mathrm{DCO}^{\mathrm{c}}$ & Multipolarity & $J_{f}^{\pi} \rightarrow J_{i}^{\pi}$ \\
\hline \multicolumn{7}{|l|}{ Band 2} \\
\hline 1775.3 & 1017.1 & 758.2 & & $0.51(25)^{1}$ & $M 1 / E 2$ & $10^{-} \rightarrow 9^{-}$ \\
\hline 1775.3 & 787.8 & 987.5 & & & & $10^{-} \rightarrow 8^{-}$ \\
\hline 2012.8 & 1209.1 & 803.7 & & & & $11^{-} \rightarrow 9^{-}$ \\
\hline 1209.1 & 519.3 & 689.8 & $<1$ & & $M 1 / E 2$ & $9^{-} \rightarrow 8^{-}$ \\
\hline 1775.3 & 787.8 & 987.5 & $<1$ & & $E 2$ & $10^{-} \rightarrow 8^{-}$ \\
\hline 1775.3 & 1017.1 & 758.2 & $4(1)$ & & $M 1 / E 2$ & $10^{-} \rightarrow 9^{-}$ \\
\hline 1775.3 & 1108.9 & 666.4 & $1(0.7)$ & & $M 1 / E 2$ & $10^{-} \rightarrow 10^{-}$ \\
\hline 2012.8 & 1775.3 & 237.5 & & & $M 1 / E 2$ & $11^{-} \rightarrow 10^{-}$ \\
\hline 2334.1 & 2012.8 & 321.3 & $1(0.6)$ & & $M 1 / E 2$ & $12^{-} \rightarrow 11^{-}$ \\
\hline 2649.4 & 2334.1 & 315.3 & & & $M 1 / E 2$ & $13^{-} \rightarrow 12^{-}$ \\
\hline 2334.1 & 1775.3 & 558.8 & $6(2)$ & $0.88(5)^{1}$ & E2 & $12^{-} \rightarrow 10^{-}$ \\
\hline 2987.9 & 2334.1 & 653.8 & $11(3)$ & $0.91(7)^{1}$ & $E 2$ & $14^{-} \rightarrow 12^{-}$ \\
\hline 3774.2 & 2987.9 & 786.3 & $21(3)$ & $0.96(2)^{1}$ & $E 2$ & $16^{-} \rightarrow 14^{-}$ \\
\hline 4517.9 & 3774.2 & 743.7 & $14(3)$ & $0.97(1)^{1}$ & $E 2$ & $18^{-} \rightarrow 16^{-}$ \\
\hline 5220.7 & 4517.9 & 702.8 & $10(2)$ & $1.00(3)^{1}$ & $E 2$ & $20^{-} \rightarrow 18^{-}$ \\
\hline 5460.9 & 5220.7 & 240.2 & $3(1)$ & $0.46(4)^{1}$ & $M 1 / E 2$ & $21^{-} \rightarrow 20^{-}$ \\
\hline 6008.8 & 5460.9 & 547.9 & $3(1)$ & $0.65(5)^{1}$ & $M 1 / E 2$ & $22^{-} \rightarrow 21^{-}$ \\
\hline 6008.8 & 5220.7 & 788.1 & $3(1)$ & & $E 2$ & $22^{-} \rightarrow 20^{-}$ \\
\hline 2649.4 & 2012.8 & 636.6 & & & $E 2$ & $13^{-} \rightarrow 11^{-}$ \\
\hline 3406.2 & 2649.4 & 756.8 & & & $E 2$ & $15^{-} \rightarrow 13^{-}$ \\
\hline 4204.9 & 3406.2 & 798.7 & & & $E 2$ & $17^{-} \rightarrow 15^{-}$ \\
\hline 1108.9 & 746.1 & 362.8 & & $1.13(12)^{2}$ & $M 1 / E 2$ & $10^{-} \rightarrow 9^{-}$ \\
\hline 1108.9 & 458.4 & 650.5 & $30(1)$ & $1.68(10)^{2}$ & $E 2$ & $10^{-} \rightarrow 8^{-}$ \\
\hline 1849.6 & 1108.9 & 740.7 & $12(2)$ & $0.91(8)^{1}, 1.89(26)^{2}$ & $E 2$ & $12^{-} \rightarrow 10^{-}$ \\
\hline 2680.2 & 1849.6 & 830.6 & $6(2)$ & $1.09(26)^{1}, 1.68(32)^{2}$ & $E 2$ & $14^{-} \rightarrow 12^{-}$ \\
\hline 3641.8 & 2680.2 & 961.6 & $5(1)$ & $1.25(35)^{1}, 2.70(37)^{2}$ & $E 2$ & $16^{-} \rightarrow 14^{-}$ \\
\hline 4605.6 & 3641.8 & 963.8 & $2(1)$ & $1.90(50)^{2}$ & $E 2$ & $18^{-} \rightarrow 16^{-}$ \\
\hline 1849.6 & 1489.4 & 360.2 & & & $M 1 / E 2$ & $12^{-} \rightarrow 11^{-}$ \\
\hline 2680.2 & 2356.0 & 324.2 & & & $M 1 / E 2$ & $14^{-} \rightarrow 13^{-}$ \\
\hline 1489.4 & 746.1 & 743.3 & $27(1)$ & $1.84(9)^{2}$ & $E 2$ & $11^{-} \rightarrow 9^{-}$ \\
\hline 2356.0 & 1489.4 & 866.6 & $11(2)$ & $1.06(8)^{1}, 1.94(14)^{2}$ & $E 2$ & $13^{-} \rightarrow 11^{-}$ \\
\hline 3342.7 & 3456 & 986.7 & $5(1)$ & $1.12(10)^{1}, 1.95(41)^{2}$ & $E 2$ & $15^{-} \rightarrow 13^{-}$ \\
\hline 4166.9 & 3342.7 & 824.2 & $1(0.5)$ & $2.25(67)^{2}$ & $E 2$ & $17^{-} \rightarrow 15^{-}$ \\
\hline 746.1 & 458.4 & 226.8 & $28(1)$ & $0.57(11)^{1}, 0.94(7)^{2}$ & $M 1 / E 2$ & $9^{-} \rightarrow 8^{-}$ \\
\hline 1244.1 & 458.4 & 724.8 & $18(2)$ & $1.57(13)^{2}$ & $E 2$ & $10^{-} \rightarrow 8^{-}$ \\
\hline 2071.5 & 1244.1 & 827.4 & $7(3)$ & $1.09(37)^{1}, 1.93(29)^{2}$ & $E 2$ & $12^{-} \rightarrow 10^{-}$ \\
\hline 3046.0 & 2071.5 & 974.5 & $3(1)$ & $1.77(51)^{2}$ & $E 2$ & $14^{-} \rightarrow 12^{-}$ \\
\hline 1244.1 & 746.1 & 498.0 & & & $M 1 / E 2$ & $10^{-} \rightarrow 9^{-}$ \\
\hline 1841.4 & 1108.9 & 732.5 & & $2.02(55)^{2}$ & $E 2$ & $12^{-} \rightarrow 10^{-}$ \\
\hline
\end{tabular}


TABLE I. (Continued.)

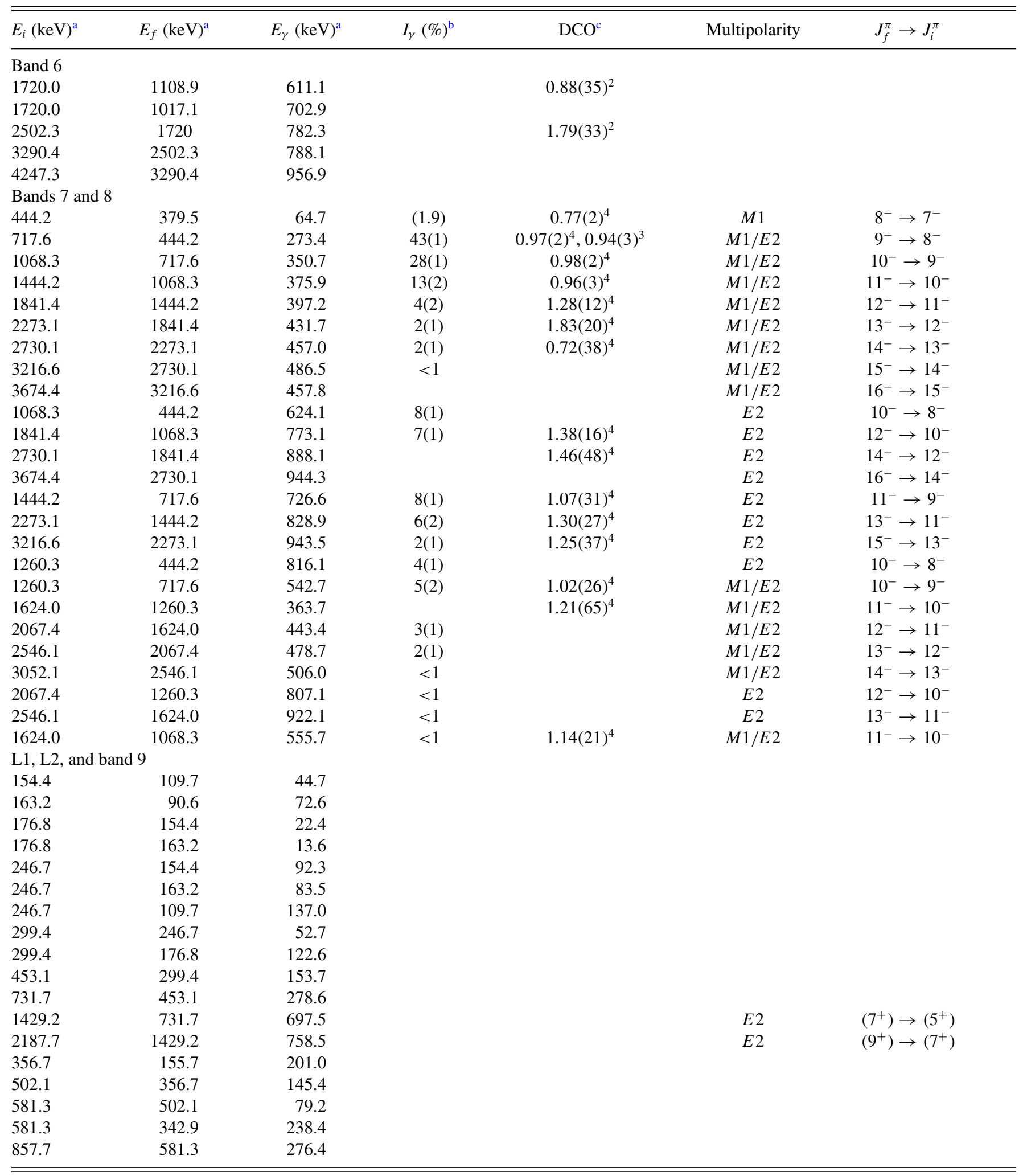

a Energies are accurate $\pm 0.3 \mathrm{keV}$.

${ }^{\mathrm{b}}$ Normalization to 100 for the $159-\mathrm{keV}$ transition.

${ }^{\mathrm{c}} 1$; stretched quadrupole gate, 2; stretched dipole gate, 3; total DCO projection, 4; 273-351 dipole sum gate. 
(a)
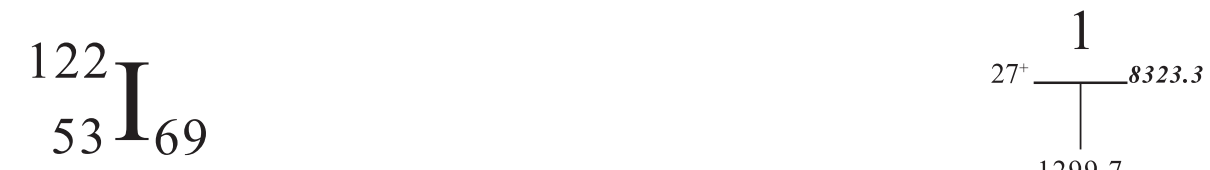

1299.7

2

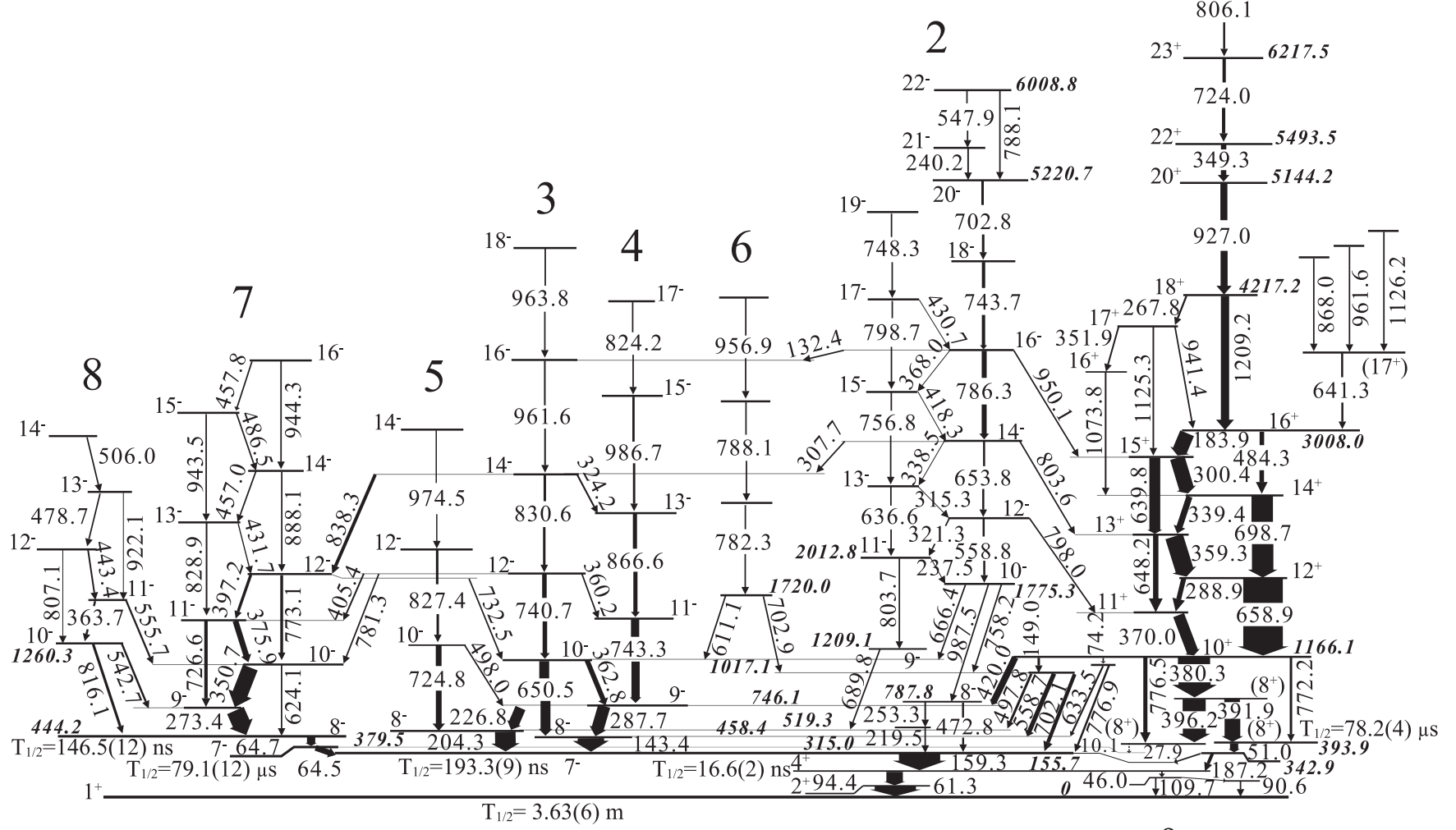

$\mathrm{T}_{1 / 2}=3.63(6) \mathrm{m}$

(b)

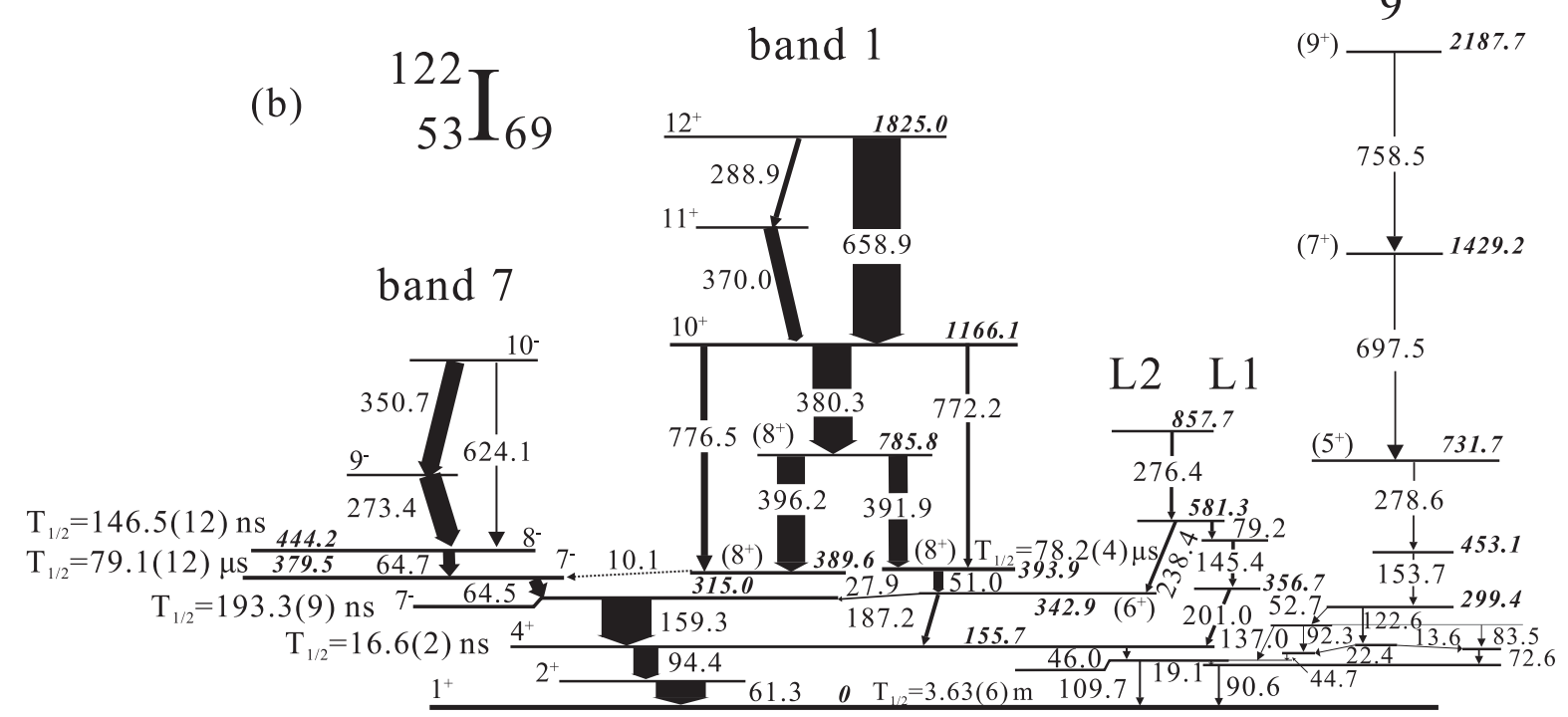

FIG. 4. Level scheme of ${ }^{122} \mathrm{I}$ deduced from the present work with the ${ }^{120} \mathrm{Sn}\left({ }^{7} \mathrm{Li}, 5 n\right){ }^{122} \mathrm{I}$ reaction at $E_{\text {lab }}=58 \mathrm{MeV}$. In panel (b), low-lying levels including the weakly populated positive-parity states are exhibited. The isomers identified in the present work are indicated by a half-life of $T_{1 / 2}$.

values suggest that the 94- and $159-\mathrm{keV}$ transitions might be of $E 2$ and $E 3$, respectively. Accordingly, the isomeric spin parity at $315 \mathrm{keV}$ can be assigned $7^{-}$.
A half-life of the $315-\mathrm{keV}$ isomer was deduced by analyzing decay curves from the beam- $\gamma$ correlations, which was defined as the $\gamma$-time conditions. The decay curves were 


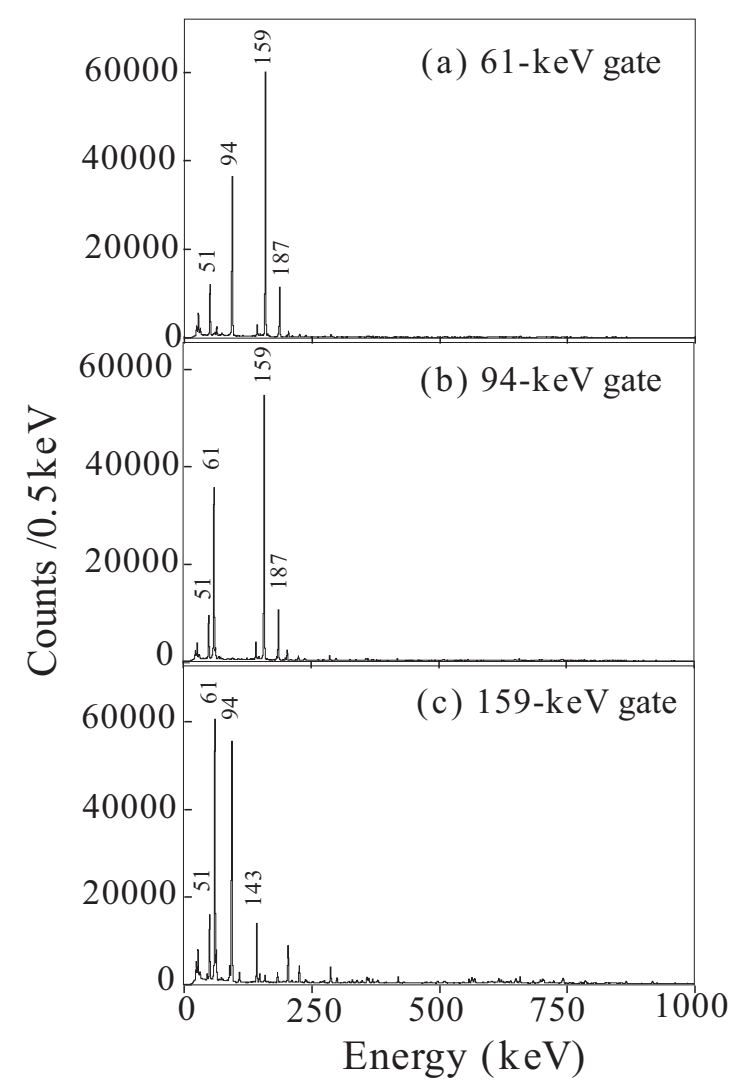

FIG. 5. Prompt coincidence $\gamma$-ray spectra by gating on (a) the 61-keV transition, (b) the $94-\mathrm{keV}$ transition, and (c) the $159-\mathrm{keV}$ transition within a time interval of $\pm 140 \mathrm{~ns}$.

obtained by gating on the 61-, 94-, and $159-\mathrm{keV}$ transitions. These curves were fitted with an exponential decay function with a constant background by using the maximum likelihood method. As shown in Fig. 7, the deduced half-lives of the decay curves from three different gate conditions were

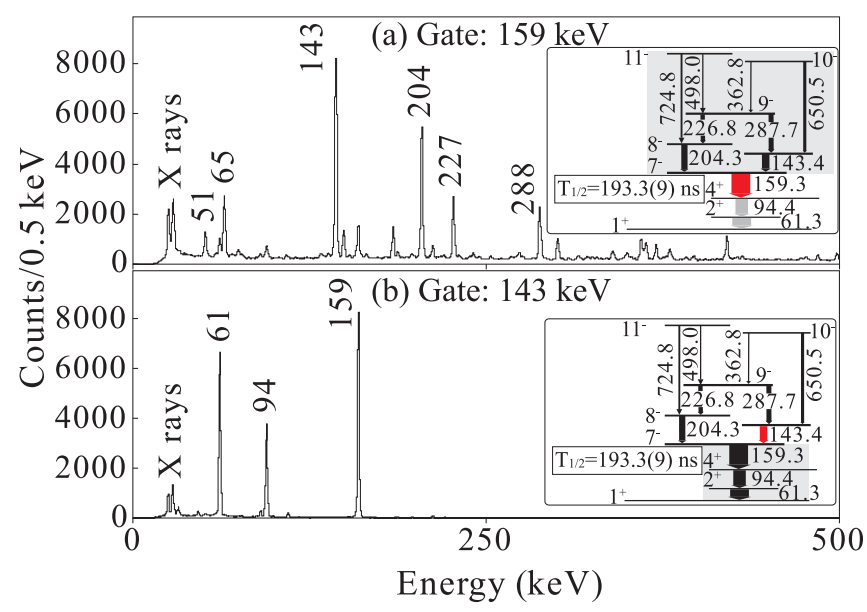

FIG. 6. Coincidence $\gamma$-ray spectra showing transitions occurring 80-900 ns before (early delayed) the gating 159-keV transition (a) and $80-900 \mathrm{~ns}$ after(late delayed) the gating $143-\mathrm{keV}$ transition (b).
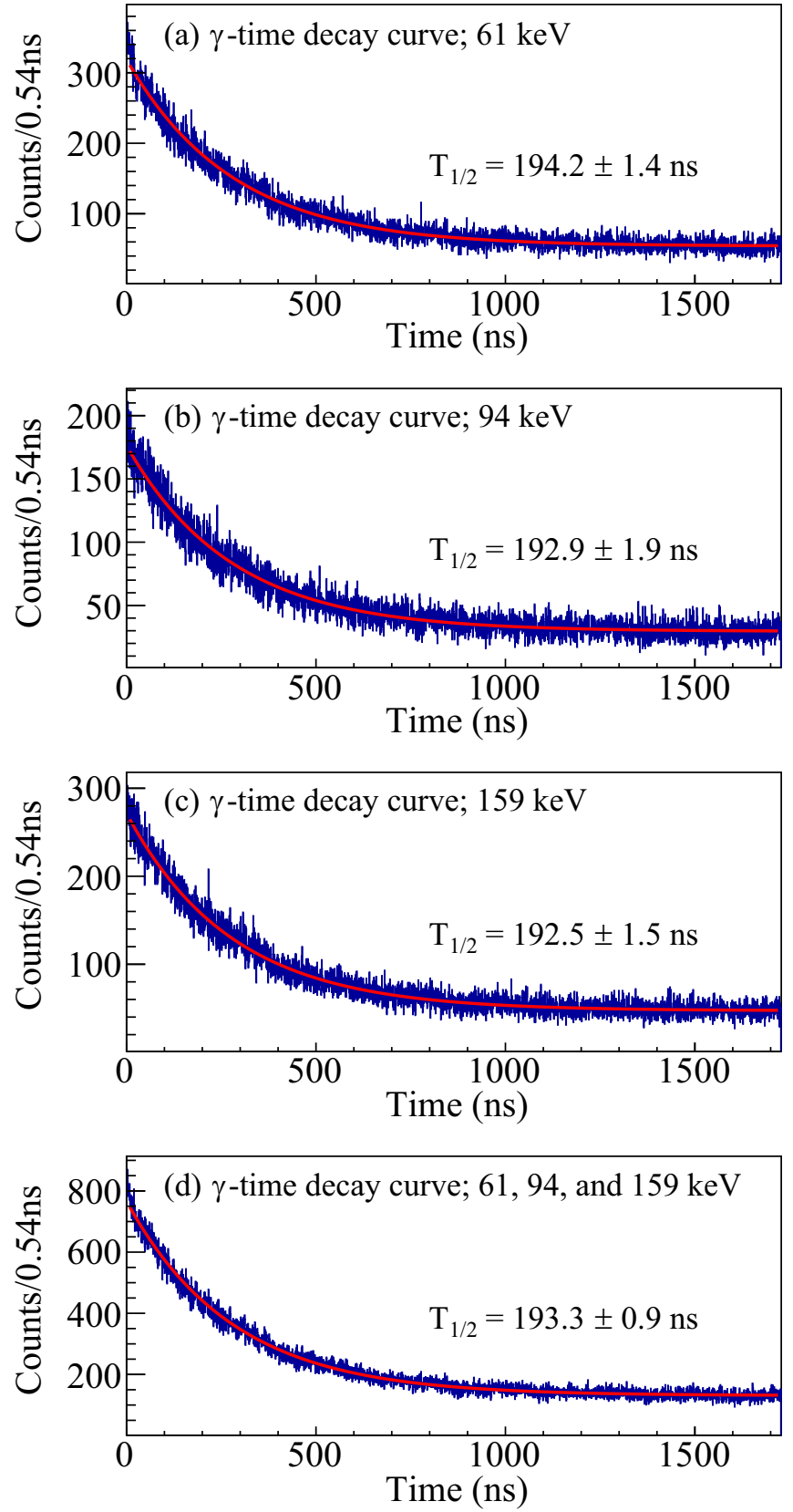

FIG. 7. Decay curves with gate on transitions of (a) 61, (b) 94 , and (c) $159 \mathrm{keV}$ following the $7^{-}$isomer at $315 \mathrm{keV}$. (d) The total decay curve with the 61-, 94-, and 159-keV transitions. The red solid lines show the fit results with half-lives as indicated.

consistent. Consequently, the half-life of the $315 \mathrm{keV}$ was determined to be 193.3(9) ns with the total decay curve obtained by three gate conditions, as shown in Fig. 7(d).

To identify isomers with a half-life of a few ns and to isolate the lifetimes of individual states between the ground state and the $315-\mathrm{keV}$ isomer, we analyzed $\gamma-\gamma$-time cube data. As already described, the time relationship between pairs of $\gamma$ rays could be obtained from such three-dimensional $\gamma_{\text {start }}-\gamma_{\text {stop }}$-time matrices by taking the background-subtracted $\gamma$-ray gates on transitions preceding (start) and following any 


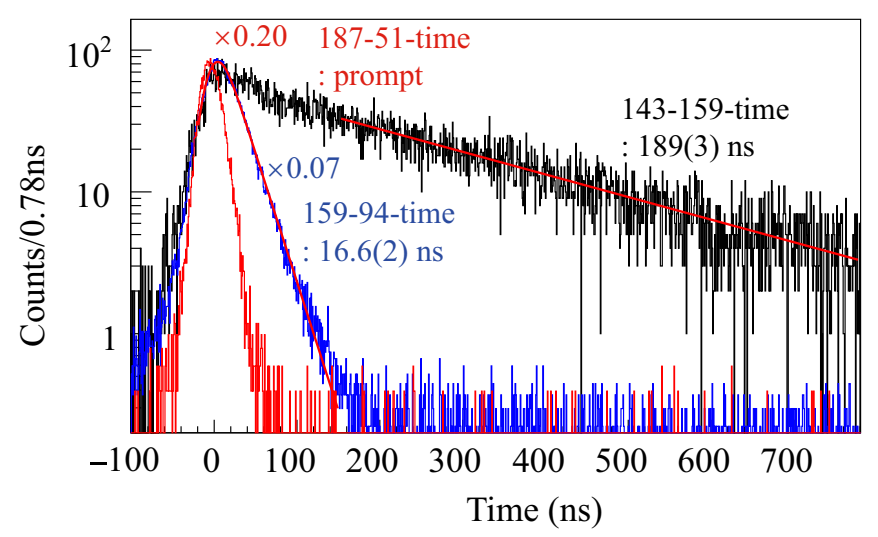

FIG. 8. $\gamma$ - $\gamma$-time spectra. The labels indicate the "start" and "stop" transitions, with a decay curve to the right of the prompt position indicating that the isomeric state is below the start transition. For the comparison, the time spectra of 187-51 keV and 159-94 $\mathrm{keV} \gamma-\gamma$ gated conditions were normalized to the time spectrum of $143-159 \mathrm{keV}$ condition with the indicated factors of 0.20 and 0.07 , respectively. Red solid lines represent the fit results with half-life values as shown.

state (stop). Figure 8 shows $\gamma-\gamma$-time spectra by setting a start gate and a stop gate between pairs of the $143-159 \mathrm{keV}, 159-94$ $\mathrm{keV}$, and $187-51 \mathrm{keV}$ transitions. The time spectrum for the $143-159 \mathrm{keV}$ indicates a decay curve for the $315-\mathrm{keV}$ isomer while the $187-51 \mathrm{keV}$ spectrum indicates a prompt time curve for the $343-\mathrm{keV}$ state. One can see that the spectrum for the 159-94 keV transition is different from that of the 187-51 keV transition; thus, the $156-\mathrm{keV}$ state should be an isomer with a half-life of a few ns. The Gaussian-exponential convoluted function with the maximum likelihood method was applied to deduce the half-life of the $159-\mathrm{keV}$ isomer from the 159-94 keV time spectrum, as shown in Fig. 8. As a result, the half-life value of 16.6(2) ns was obtained. On the other hand, the half-life of the $315-\mathrm{keV}$ isomer could also be obtained by fitting the tail of the time spectrum with a function composed of an exponential decay function and a constant background. The deduced half-life value of 188(3) ns is consistent with the evaluated half-life value of 193.3(9) ns within the uncertainties.

As shown in Fig. 4(b), the 91- and 110-keV levels are additional decaying paths between the $315-\mathrm{keV}$ state and the ground state. On the basis of these two levels, several states, denoted by $\mathrm{L} 1$ including band 9 , are weakly populated. We assigned this bandhead at $732 \mathrm{keV}$ as $5^{+}$according to the observation of the similar bands in ${ }^{124} \mathrm{I}$ [9] and ${ }^{126} \mathrm{I}$ [9]. Another weak populated level, L2, is also likely positive parity in nature, but spin-parity assignment could not be made.

For the level scheme of ${ }^{122} \mathrm{I}$, it should be noticed that both the $7^{-}$level at $380 \mathrm{keV}$ and the $8^{-}$level at $444 \mathrm{keV}$ are isomers and the individual decaying transition from them is a doublet of $65-\mathrm{keV} \gamma$ rays. In the $1 \mathrm{~ns}-1.7 \mu$ s data, these doublet transitions were not correlated in prompt coincidence. Instead, from delayed coincidence with the transitions above and below the isomers, a doublet could be isolated and the $380-\mathrm{keV}$ state could be considered to be a long-lived isomer

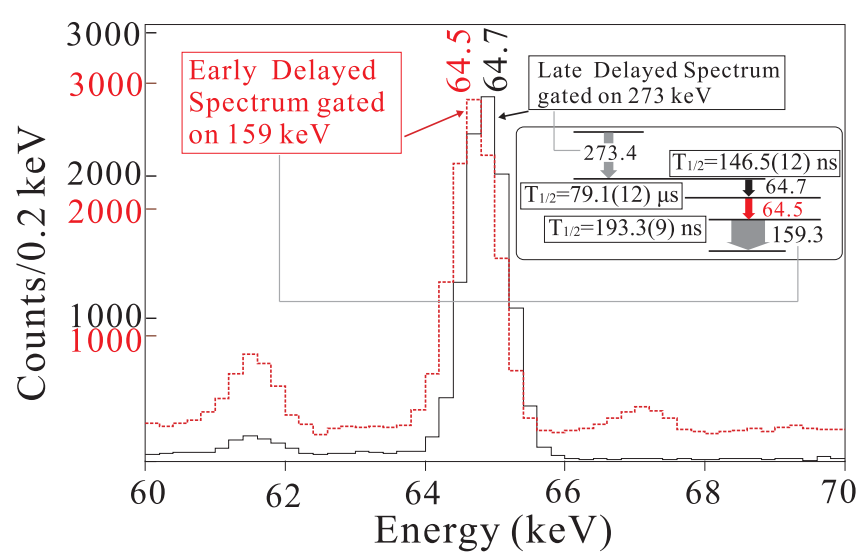

FIG. 9. Energy difference of a doublet $65-\mathrm{keV} \gamma$ rays between the early-delayed spectrum by gating on the $159-\mathrm{keV}$ transition and the late-delayed spectrum by gating on the $273-\mathrm{keV}$ transition. Also see Fig. 4.

with a few tens $\mu$ s. Figure 9 shows $65-\mathrm{keV}$ double spectra from an early delayed coincidence by gating on the $159-\mathrm{keV}$ transition and a late delayed coincidence by gating on the 273$\mathrm{keV}$ transition. One can see that a doublet could apparently be split by the mean values of 64.5 and $64.7 \mathrm{keV}$, respectively. Here the $64.5-\mathrm{keV} \gamma$-ray corresponds to the transition feeding the $315-\mathrm{keV}$ isomer $(193.3 \mathrm{~ns}$ ) and the $64.7-\mathrm{keV} \gamma$-ray is the transition depopulating the 444-keV isomer (146.5 ns).

Figure 10(a) shows a decay curve, obtained from the $\gamma$ time data, along with a fit indicating the half-life of 146.5(12) ns for the 64.7-keV transition. In contrast, as shown in Fig. 10(b), the half-life of the $7^{-}$isomer was found to be
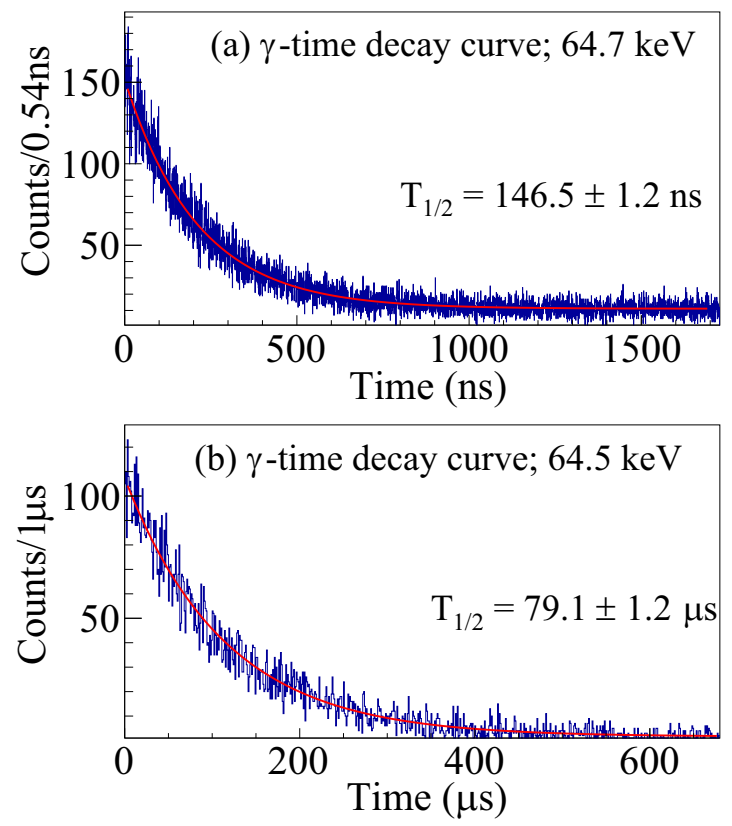

FIG. 10. (a) Decay curve for the 64.7-keV transition showing a half-life of 146.5(12) ns. A beam cycle is $1 \mathrm{~ns}$ on and $1.7 \mu$ s off. (b) Decay curve for the $64.5 \mathrm{keV}$ transition showing a half-life of $79.1(12) \mu \mathrm{s}$. A beam cycle is $60 \mu \mathrm{s}$ on and $880 \mu \mathrm{s}$ off. 

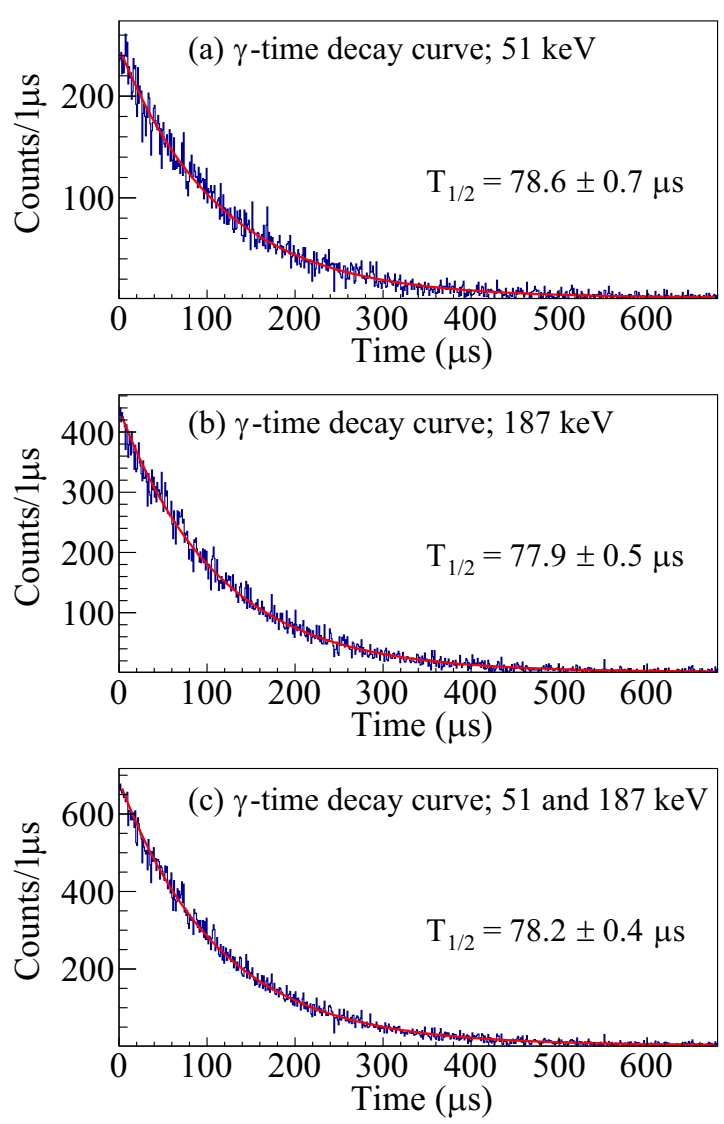

FIG. 11. Decay curves for (a) the 51-keV transition, (b) the 187$\mathrm{keV}$ transition, and (c) both transitions. The fit results are shown in red solid lines and respective half-life values are indicated.

79.1(12) $\mu \mathrm{s}$. This result was obtained from the second experiment with a beam cycle of $60 \mu \mathrm{s}$ on and $880 \mu$ s off by setting gates on the delayed $64.5-\mathrm{keV}$ transition. In addition to the $7^{-}$ isomer at $380 \mathrm{keV}$, another long-lived isomer at $394 \mathrm{keV}$ was identified from the $60 \mu \mathrm{s}$ on and $880 \mu \mathrm{s}$ off data. As shown in Fig. 11, the half-life for the 394-keV isomer was measured to be 78.2(4) $\mu$ s with the total decay curve obtained by the gate conditions of 51 and $187 \mathrm{keV}$. As was pointed out earlier, the present result explains why two independent-transition paths feeding the isomer were observed in the previous work [16].

Figure 12 provides further evidence of the existence of two isomeric states. Although the $65-\mathrm{keV}$ transition and the 51-keV transition are from different isomers, they both feed the $315-\mathrm{keV}$ isomer. The $51-\mathrm{keV}$ transition, depopulating the $394-\mathrm{keV}$ isomer, connects the $315-\mathrm{keV}$ isomer through the $28-\mathrm{keV}$ transition. The $\gamma-\gamma$ and $\gamma$-time relations imply that the $8^{+}$state at $390 \mathrm{keV}$ might predominantly depopulate to the $7^{-}$isomer at $380 \mathrm{keV}$ via an unobserved $10-\mathrm{keV}$ transition. See Figs. 6 and 13 where there are no correlations between the 396-keV transition feeding the 390-keV level and the 159-94-61 keV transitions. As far as our overall data analyses are concerned, we could not find a direct connection between the two isomers with the identical lifetime.

Let us explain more details regarding the existence of the triple $8^{+}$states at 786,390 , and $394 \mathrm{keV}$. The $390-\mathrm{keV}$ level fed by both the 396- and 776-keV transitions has the following

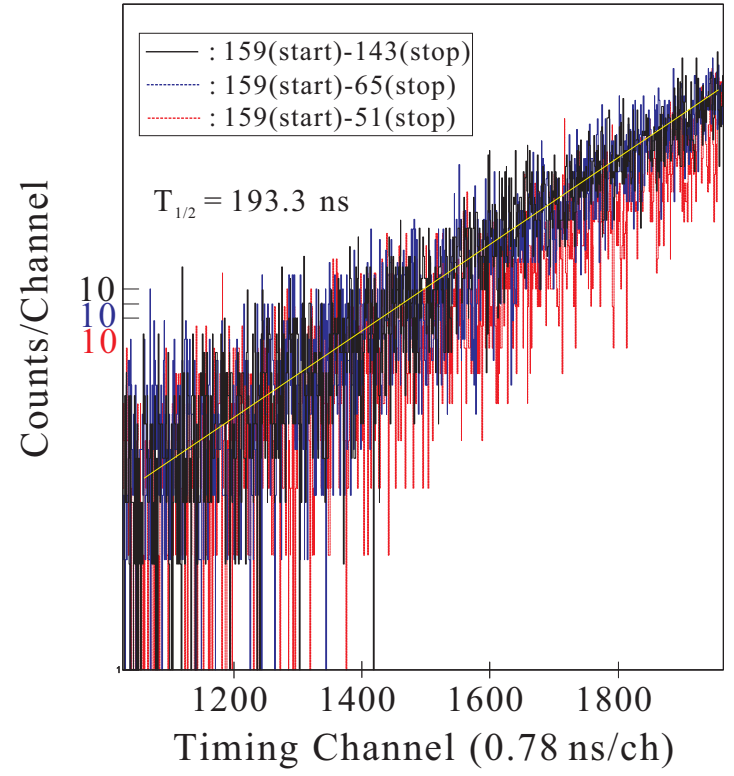

FIG. 12. $\gamma-\gamma$-time spectra showing the lifetime of the $315-\mathrm{keV}$ isomer when gating on the starting $159-\mathrm{keV}$ transition and the stopping 143-, 65-, and 51-keV transitions. The three transitions feed all together the $315-\mathrm{keV}$ isomer from which the $159-\mathrm{keV}$ transition decays. The yellow (gray) solid line represents the guiding decay curve with the fixed half-life value of $193.3 \mathrm{~ns}$, determined by the present work. See Figs. 5 and 7.

features: First, there is no observation of transitions depopulating this level. Second, there is no sign indicating an isomer comparable with the observed isomers. According to DCO ratios, the 380- and the 396-keV transitions and their cross-over transition, $776 \mathrm{keV}$, indicate a stretched quadrupole structure. Moreover, the 392-keV transition, which depopulates parallel with the 396-keV transition, the $786-\mathrm{keV}$ level fed by the $380-\mathrm{keV}$ transition, and the cross-over $772-\mathrm{keV}$ transition also have a stretched quadrupole nature. Further, the $380-\mathrm{keV}$ transition shows the same character as the $E 2$ quadrupole transitions. This implies that the $786-\mathrm{keV}$ level should be $8^{+}$rather than $9^{+}$. If the spin parity of the $786-\mathrm{keV}$ level is $9^{+}$, then all the 380-, 396-, and 392-keV transitions should

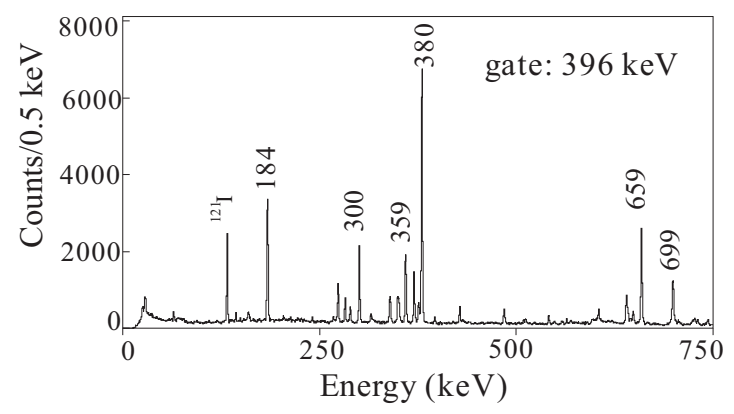

FIG. 13. Prompt coincidence $\gamma$-ray spectrum by gating on the $396-\mathrm{keV}$ transition within a time interval of $\pm 140 \mathrm{~ns}$. Notice that no $\gamma$-ray transitions below the $390-\mathrm{keV}$ level, such as $159-, 94-$, and $61-\mathrm{keV}$ ones, are observed. See also Fig. 5 where spectra gated on 159,94 , and $61 \mathrm{keV}$ show no peaks at $396 \mathrm{keV}$. 

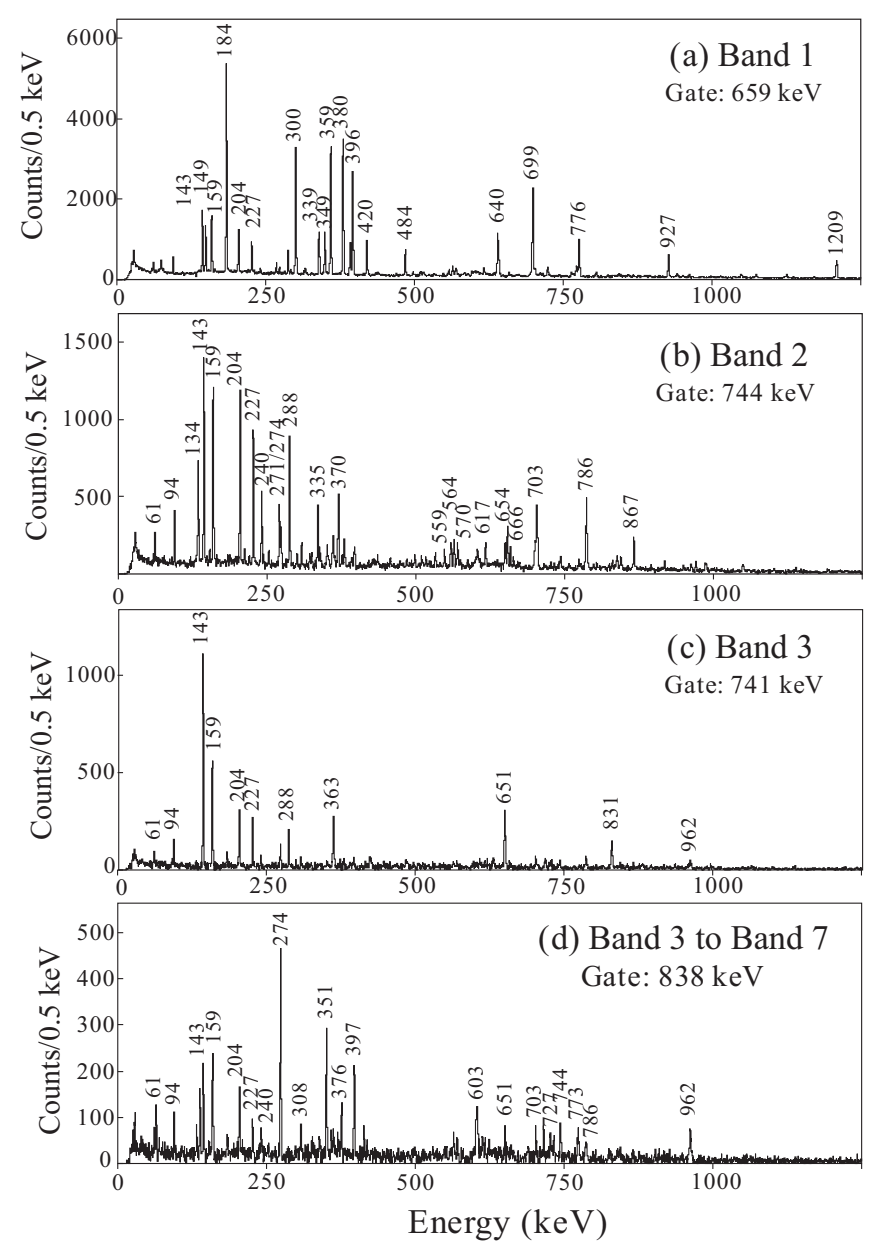

FIG. 14. $\gamma$-ray transitions in and between the collective bands in ${ }^{122} \mathrm{I}$. (a) $\gamma$ rays in coincidence with the $659-\mathrm{keV}$ transition belong to band 1 . (b) $\gamma$ rays in coincidence with the $744-\mathrm{keV}$ transition belong to band 2. (c) $\gamma$ rays in coincidence with the $741-\mathrm{keV}$ transition belong to band 3. (d) $\gamma$ rays in coincidence with the $838-\mathrm{keV}$ transition which connects band 3 to band 7 . Notice that the connecting transitions between bands 3 and 7 complete the excitation energies, leading to the bandhead energy at $444.2 \mathrm{keV}$ for band 7 .

be $M 1 / E 2$ having a dominating dipole nature as opposed to quadrupole. According to the DCO ratio values, this is not the case. It is worthwhile to know that all the $M 1 / E 2$ transitions follow the dipole-dominating character as shown in Table I. Interestingly], the DCO values for the corresponding three transitions indicate a quadrupole character [14]. Nevertheless, the 380-, 396-, and 392-keV peaks were assigned as $M 1 / E 2$ dipole transitions [14]. Such assignments may be based on the simple assumption that the 380-keV, 396-, and 392-keV transitions would be of $M 1 / E 2$ dipole cascades while the 776and $772-\mathrm{keV}$ transitions are the cross-over $E 2$ transitions.

Figure 14 shows the $\gamma$-ray transitions assigned to ${ }^{122} \mathrm{I}$. Compared to our previous report in Ref. [9], the signature partner of bands 2 and 9 are newly added. Our result on the observation of collective bands except for band 9 coincides with the result by Singh et al. [12]. It should be noticed that the excitation energies in Ref. [12] were based on our result.
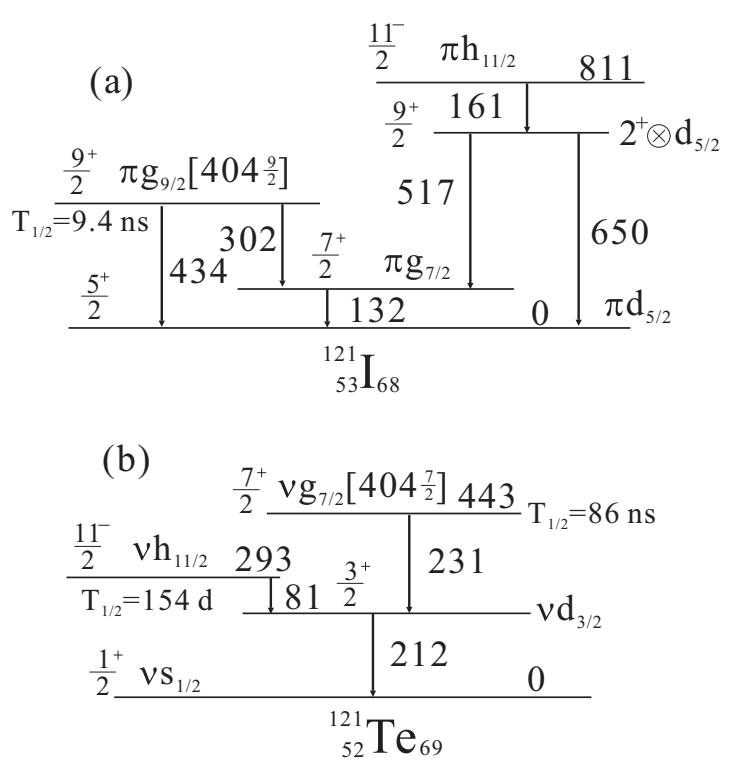

FIG. 15. Low-lying states in ${ }^{121} \mathrm{I}$ (a) and in ${ }^{121} \mathrm{Te}$ (b). The Nilsson configurations for the high- $K$ orbitals are denoted. Energies are given in keV. Data are taken from the National Nuclear Data Center [17].

\section{DISCUSSION}

\section{A. Low-lying states}

For interpreting the low-lying shell structure of ${ }^{122} \mathrm{I}$, we begin with a comparison of low-lying levels in the neighboring isotope and isotone. Figure 15 shows the low-lying states observed in ${ }^{121} \mathrm{I}$ and ${ }^{121} \mathrm{Te}$, respectively. From this comparison, the expected proton and neutron configurations for ${ }^{122} \mathrm{I}$ are $\pi d_{5 / 2} \nu s_{1 / 2}, \pi d_{5 / 2} \nu d_{3 / 2}, \pi g_{7 / 2} \nu s_{1 / 2}, \pi g_{7 / 2} \nu d_{3 / 2}$, and $\pi h_{11 / 2} v h_{11 / 2}$ for positive-parity states and $\pi d_{5 / 2} v h_{11 / 2}$, $\pi g_{7 / 2} v h_{11 / 2}$, and $\pi g_{9 / 2} v h_{11 / 2}$ for negative-parity states. As was mentioned earlier, the $\pi h_{11 / 2} v h_{11 / 2}$ configuration is responsible for the $10^{+}$state on which band 1 is built. Notice that the sum excitation energy of the $\pi h_{11 / 2}$ bandhead in ${ }^{121} \mathrm{I}$ and the $v h_{11 / 2}$ one in ${ }^{121} \mathrm{Te}, 1104 \mathrm{keV}$, is almost identical to the observed $1166 \mathrm{keV}$ at $10^{+}$. Based on the above comparison, the most probable configuration for the ground state is the $\pi d_{5 / 2} \nu s_{1 / 2}$ configuration. However, this configuration is not feasible to reproduce the spin parity of the $1^{+}$ground state. Once we look closely at the $\beta$-decay scheme from ${ }^{122} \mathrm{Xe}$, the $\pi d_{5 / 2} \nu d_{3 / 2}$ configuration should be more compatible with the ground state for ${ }^{122} \mathrm{I}$. This argument is based on the so-called Gamow-Teller transition related to an allowed transition by a transformation between the $\pi d_{5 / 2}$ orbital in ${ }^{122} \mathrm{Xe}$ and the $v d_{3 / 2}$ orbital in ${ }^{122} \mathrm{I}$. Accordingly, the ground-state structure with the spin parity of $1^{+}$should mainly be associated with the $\pi d_{5 / 2} \nu d_{3 / 2}$ configuration. This conclusion is in good agreement with the theoretical predictions by shell model calculations.

In order to explain the underlying properties of the nuclear structure in low-lying states, we performed theoretical calculations with a large-scale spherical shell model in the space of $50<Z, N<82$. The charge-dependent Bonn potential we used is based on the realistic Sn100 Hamiltonian [18] in this model space. For the global description of the nuclei in 
this region, the proton-proton and neutron-neutron parts of the Hamiltonian were modified with the scaling factors of 1.03 and 0.892 , respectively. The calculations were carried out using the shell model code KSHELL [19]. The dimensions of matrices generated for ${ }^{122} \mathrm{I}$ with 19 neutrons outside the $N=50$ shell closure in the unconstrained space of the $g_{7 / 2}$, $d_{5 / 2}, d_{3 / 2}, s_{1 / 2}$, and $h_{11 / 2}$ orbitals are too large for practical calculations. Hence, it is necessary to diminish this large space by truncations. We imposed the restricted active space that is characterized by having neutrons excited from the constrained $g_{7 / 2}$ and $d_{5 / 2}$ subspace into the unconstrained upper $d_{3 / 2}$, $s_{1 / 2}$, and $h_{11 / 2}$ subspace. With this constraint, we calculated two cases that five or seven neutrons distribute freely in the $d_{3 / 2}, s_{1 / 2}$, and $h_{11 / 2}$ subspace while three free protons are in the full space. Each subspace-constrained calculation fixed the rest of the neutrons in the $\left(g_{7 / 2}\right)^{8}\left(d_{5 / 2}\right)^{6}$ or $\left(g_{7 / 2}\right)^{8}\left(d_{5 / 2}\right)^{4}$ configuration. Thus, these model subspaces are respectively abbreviated as the " $N 5$ " and " $N 7$ " subspaces hereafter, for convenience. It should be emphasized that the description on the configuration will be interpreted only with free valence nucleons, e.g., $\pi\left[\left(g_{7 / 2}\right)^{2}\left(d_{5 / 2}\right)^{1}\right] v\left[\left(d_{3 / 2}\right)^{1}\left(h_{11 / 2}\right)^{4}\right]$ instead of $\pi\left[\left(g_{7 / 2}\right)^{2}\left(d_{5 / 2}\right)^{1}\right] v\left[\left(g_{7 / 2}\right)^{8}\left(d_{5 / 2}\right)^{6}\left(d_{3 / 2}\right)^{1}\left(h_{11 / 2}\right)^{4}\right]$ for the $N 5$ subspace calculation. The results with respect to the energy versus spin are displayed in Fig. 16.

It is found that the N5 subspace calculation is more favorable to reproduce low-lying levels. The positive-parity states based on the $N 7$ subspace calculation are lying in higher energies by about $500 \mathrm{keV}$ than those based on the $N 5$ subspace calculation results. Such shifts are found to be attributed to the different components in the proton $g_{7 / 2}$ and $d_{5 / 2}$ orbitals. For instance, the $N 5$ subspace calculation shows a tendency to organize in the $\pi\left(g_{7 / 2}\right)^{2}\left(d_{5 / 2}\right)^{1}$ configuration while the $N 7$ subspace calculation tends to produce the $\pi\left(g_{7 / 2}\right)^{3}$ configuration. Consequently, the $\pi d_{5 / 2} \nu d_{3 / 2}$ configuration is most likely responsible for producing the favored low-lying positive states with spin parities from $1^{+}$to $4^{+}$. As shown in Fig. 16(a), the result for these states reasonably matches the experimental data although the $1^{+}$level could not be reproduced at 0 but instead is predicted at the energy of $72 \mathrm{keV}$. To the states above $J^{\pi}=4^{+}$, the $\pi\left(g_{7 / 2}\right)^{2}\left(d_{5 / 2}\right)^{1}$ and the $\pi\left(g_{7 / 2}\right)^{3}$ configurations comparably contribute. For example, the $\pi\left(g_{7 / 2}\right)^{2}\left(d_{5 / 2}\right)^{1}$ configuration tends to produce the positive states with the even spin numbers of 6 and 8 while the $\pi\left(g_{7 / 2}\right)^{3}$ configuration tends to produce the positive states with odd spin numbers of 5 and 7.

We notice that the $8^{+}$and $10^{+}$states appear to be predominated by the $\pi\left[\left(g_{7 / 2}\right)^{2}\left(d_{5 / 2}\right)^{1}\right] \nu\left[\left(d_{3 / 2}\right)^{1}\left(h_{11 / 2}\right)^{4}\right]$ configuration. For the $8^{+}$state, the $\pi\left(d_{5 / 2}\right)^{1} v\left(d_{3 / 2}\right)^{1}\left(h_{11 / 2}\right)^{2}$ configuration with the amplitude of $28 \%$ is responsible to reproduce the spin parity as indicating spin alignments of $j_{p}=5 / 2^{+}$and $j_{n}=11 / 2^{+}$. The total $11 / 2^{+}$spin of the neutrons is from the contributions of the $3 / 2^{+}$and $4^{+}$spin parities due to the $\left(d_{3 / 2}\right)^{1}$ and $\left(h_{11 / 2}\right)^{2}$ occupancies. Similarly, a favored $10^{+}$state is predicted at $934 \mathrm{keV}$ mainly by $\pi\left(d_{5 / 2}\right)^{1} v\left(d_{3 / 2}\right)^{1}\left(h_{11 / 2}\right)^{2}$ with the amplitude of $27 \%$. The $8^{+}$ state at $786 \mathrm{keV}$, as shown in Fig. 16(a), is consistent with the predicted $8^{+}$state. However, spin states higher than $8^{+}$ based on the $\pi\left(d_{5 / 2}\right)^{1} v\left(d_{3 / 2}\right)^{1}\left(h_{11 / 2}\right)^{2}$ configuration were not observed since they are likely produced above the yrast line.
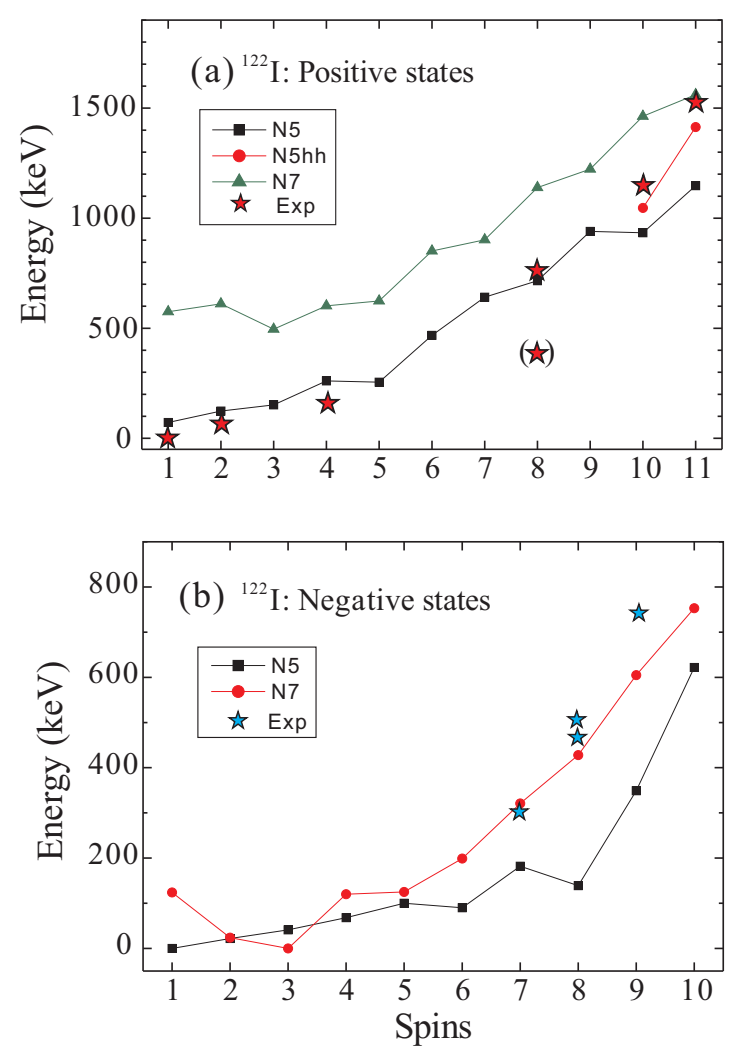

FIG. 16. Calculated single-particle energies as a function of the spins based on the large-scale shell model with the Sn100 Hamiltonian for ${ }^{122}$ I. For comparison, experimental data are included: the yrast positive-parity states (filled stars with red color) and the negative-parity states (filled stars with blue). The truncated conditions of the 19 valence neutrons are denoted by $N 5$ and $N 7$, which are five neutrons and seven neutrons freely distributed, respectively in the $d_{3 / 2}, s_{1 / 2}$, and $h_{11 / 2}$ space. The $N 5 h h$ condition denotes the $\pi h_{11 / 2} v h_{11 / 2}$ excitation under the $N 5$ subspace calculation.

This $8^{+}$state might be interpreted as being associated with the $\pi h_{11 / 2} v h_{11 / 2}$ configuration. In ${ }^{118} \mathrm{I}$ [20] and ${ }^{120} \mathrm{I}$ [21], the $8^{+}$ and $9^{+}$states were found to be closely connected to the $10^{+}$ state with the $\pi h_{11 / 2} \nu h_{11 / 2}$ configuration through $M 1$ and $E 2$ transitions. However, the $8^{+}$state at $786 \mathrm{keV}$ in ${ }^{122}$ I differs significantly in transitional structure from those in ${ }^{120} \mathrm{I}$ and ${ }^{118} \mathrm{I}$.

The present shell model successfully predicts the $10^{+}$and $11^{+}$states associated with the $\pi h_{11 / 2} v h_{11 / 2}$ configuration, which are denoted by the $N 5 h h$ subspace model in Fig. 16(a). The $10^{+}$and $11^{+}$states were predicted at 1047 and 1414 $\mathrm{keV}$, respectively, and are consistent with the experimental data. For the case of the $10^{+}$state, the wave function is dominantly composed of the $\pi\left[\left(g_{7 / 2}\right)^{2}\left(h_{11 / 2}\right)^{1}\right] \nu\left[\left(d_{3 / 2}\right)^{2}\left(h_{11 / 2}\right)^{3}\right]$ and $\pi\left[\left(g_{7 / 2}\right)^{2}\left(h_{11 / 2}\right)^{1}\right] \nu\left[\left(h_{11 / 2}\right)^{5}\right]$ configurations with the amplitudes of $19 \%$ and $11 \%$, respectively.

For the negative states, the $N 5$ and $N 7$ subspace calculations are comparable for reproducing the $1^{-}$to $5^{-}$ states. However, the favored configurations are different that a leading component for the N5 subspace calculation is the $\pi g_{7 / 2} \nu h_{11 / 2}$ configuration while it is the $\pi d_{5 / 2} \nu h_{11 / 2}$ 
configuration for the $N 7$ subspace calculation. Above the $5^{-}$ state, the experimental results follow the predictions based on the $N 7$ subspace calculation though the yrast states in this region are produced by the $N 5$ subspace calculation. Interestingly, for the $7^{-}$state, the $N 5$ subspace calculation tends to result in the $\pi d_{5 / 2} v h_{11 / 2}$ configuration. This result implies that the $7^{-}$isomer at $315 \mathrm{keV}$ is most likely based on the $\pi d_{5 / 2} v h_{11 / 2}$ configuration. The very similar features between the $\pi g_{7 / 2} v h_{11 / 2}$ and the $\pi d_{5 / 2} v h_{11 / 2}$ configurations are mainly due to the close separation of the $g_{7 / 2}$ and $d_{5 / 2}$ orbitals. These two close orbitals are readily mixed even in a small deformation. We will discuss these two orbital trajectories in more detail using a deformed shell model.

Now we turn our attention to the $7^{-}$isomer at $380 \mathrm{keV}$ and the $8^{+}$isomer at $394 \mathrm{keV}$. These unusual low-lying energy levels cannot be reproduced by the spherical shellmodel calculation within the $50<Z, N<82$ space. This unpredictability is largely due to there being no inclusion of the proton-hole $g_{9 / 2}$ orbital below the $Z=50$ shell closure. For describing the effect of the $g_{9 / 2}$ orbital, we apply a deformed shell model based on the Nilsson orbital [22,23]. The Nilsson-BCS with pairing correlation calculations define a deformed quasiparticle basis including an asymptotic quantum number, $\left[N, n_{z}, \Lambda\right] \Omega$. The quasiparticle energies were obtained by summing their respective energies given by $E_{x}=$ $\sqrt{(E-\lambda)^{2}+\Delta^{2}}$, where $E$ is the single proton or neutron energy from the Hartree-Fock calculations, $\lambda$ is the Fermi level, and $\Delta$ is the proton or neutron pairing strength parameter. The pairing parameters $\Delta_{p}=1.19 \mathrm{MeV}$ for protons and $\Delta_{n}=1.33 \mathrm{MeV}$ for neutrons were deduced from the recent atomic mass evaluation [24]. According to the total energy (Routhian) surface $[25,26]$ calculations for ${ }^{122} \mathrm{I}$, the corresponding coupled configurations built on a proton and a neutron near the Fermi surfaces reveal a weak deformed shape, indicating the quadrupole deformation $\epsilon_{2}=0.11$ to 0.16 , with a large amount of $\gamma$ softness. We present the results for the Nilsson model calculations in Fig. 17 as a function of the quadrupole deformation, $\epsilon_{2}$. For adapting the $\gamma$-soft deformation, we also investigated the Nilsson orbitals at nonaxial deformations. Figure 18 shows the associated trajectories at $\epsilon_{2}=0.16$ with respect to the $\gamma$ deformation in the $0^{\circ} \leqslant \gamma \leqslant 60^{\circ}$ region.

Around small deformations at $\epsilon_{2}=0.1$ to 0.16 , the lowlying states are dominated by the $g_{7 / 2}[422] 3 / 2, d_{5 / 2}[420] 1 / 2$, and $g_{7 / 2}[440] 1 / 2$ orbitals for protons and the $d_{3 / 2}[411] 1 / 2$, $h_{11 / 2}[523] 7 / 2, h_{11 / 2}[532] 5 / 2$, and $g_{7 / 2}[404] 7 / 2$ orbitals for neutrons. Hence, the low-lying positive states including the ground $1^{+}$state are built mainly from combinations of $\pi[422] 3 / 2 v[411] 1 / 2$ and $\pi[420] 1 / 2 v[411] 1 / 2$. In contrast, the observed negative-parity levels are most likely associated with a proton in either [422]3/2 or [420]1/2 coupled to a neutron in [532]5/2 or [523]7/2. Both the proton $g_{9 / 2}[404] 9 / 2$ and the neutron $g_{7 / 2}[404] 7 / 2$ states rapidly reduce in energy with increasing $\epsilon_{2}$ parameter, as shown by $\pi[404] 9 / 2$ minimized around $\epsilon_{2}=0.24$ and $\nu[404] 7 / 2$ minimized around $\epsilon_{2}=0.16$, respectively. Moreover, the $v[404] 7 / 2$ orbital keeps its minimum even up to $\gamma=30^{\circ}$. In contrast, the $\pi$ [404]9/2 orbital favors an axial shape with $\gamma=0^{\circ}$.
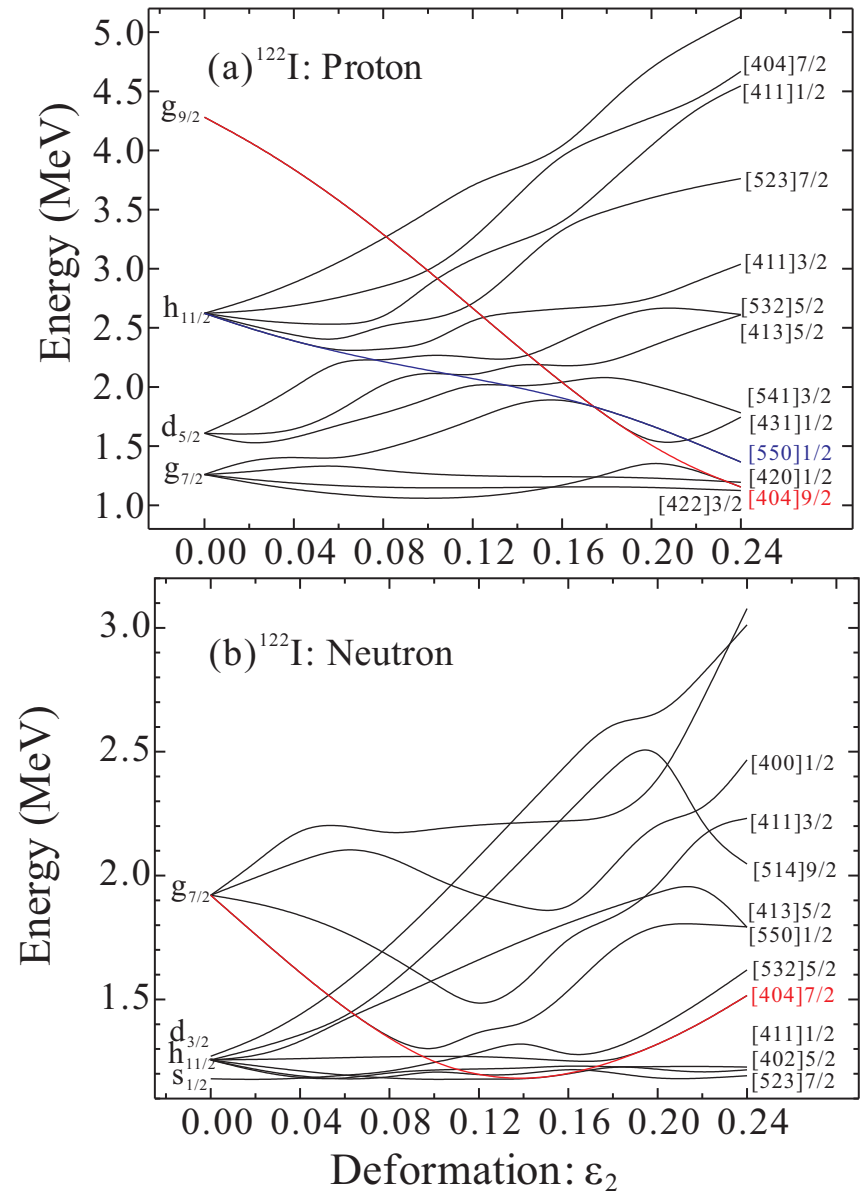

FIG. 17. Calculated quasiparticle energy diagrams as a function of quadrupole deformation parameter $\epsilon_{2}$ for (a) protons and (b) neutrons in ${ }^{122} I$, based on the Nilsson model. The used pairing parameters are $\Delta_{p}=1.19 \mathrm{MeV}$ for quasiprotons and $\Delta_{n}=1.33 \mathrm{MeV}$ for quasineutrons. On the right hand side are the low-lying levels at $\epsilon_{2}=0.24$, with the asymptotic quantum numbers $\left[N, n_{z}, \Lambda\right] \Omega$. For comparison, the spherical orbitals are also denoted at $\epsilon_{2}=0$.

The $7^{-}$isomer at $380 \mathrm{keV}$ is associated with the $\pi g_{9 / 2}[404] 9 / 2 v h_{11 / 2}[532] 5 / 2$ configuration. The situation when the odd proton and the odd neutron occupy high$\Omega$ orbitals of the intruder subshells is particularly interesting, because it leads to high- $K$ states at low-lying energies that generate some hindrance for decays ( $K$ trap). These $K$ isomers are systematically observed at $J^{\pi}=7^{-}$based on the proton-neutron $\pi g_{9 / 2}[404] 9 / 2 v h_{11 / 2}[532] 5 / 2$ configuration for ${ }^{116-122} \mathrm{I}$ [11]. The isomerism for these states comes from the forbidden nature of the decays between the $J^{\pi}=$ $7^{-}$with $K^{\pi}=7^{-}$and the $J^{\pi}=7^{-}$with $K^{\pi}=3^{-}$based on the $\pi d_{5 / 2}[431] 1 / 2 v h_{11 / 2}[532] 5 / 2$ configuration. Moreover, the $\pi g_{9 / 2}[404] 9 / 2 v h_{11 / 2}[523] 7 / 2$ configuration generates the $K^{\pi}=8^{-}$isomer at $444 \mathrm{keV}$. We notice that for ${ }^{120} \mathrm{I}$, the corresponding $7^{-}$state leads to the $\beta$-decaying isomer lying very close to the ground state [11]. In contrast, the ${ }^{124}$ I has an isomer at $8^{-}$with $T_{1 / 2}=14 \mathrm{~ns}$ rather than at $7^{-}$[11].

The $8^{+}$state at $394 \mathrm{keV}$ could be explained by a coupled configuration of a proton hole in the $g_{9 / 2}$ orbital 


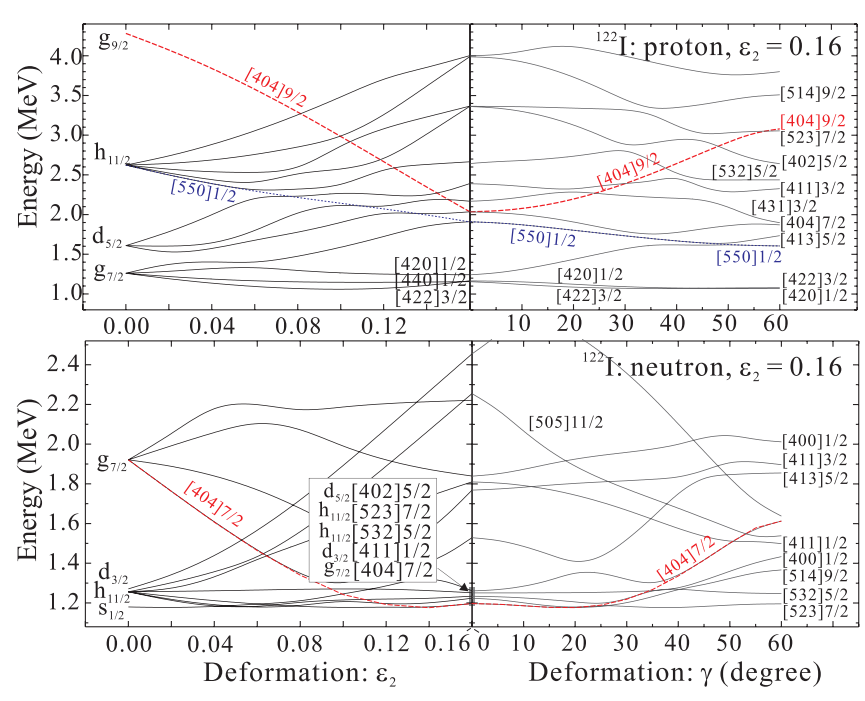

FIG. 18. Nilsson trajectories in axial deformations over $0 \leqslant$ $\epsilon_{2} \leqslant 0.16$ in left panel and in nonaxial deformations at $\epsilon_{2}=0.16$ over $0^{\circ} \leqslant \gamma \leqslant 60^{\circ}$. For the proton hole [404]9/2 and the neutron hole [404]7/2 orbitals, their trajectories are denoted in red (dashed) lines. The blue (dotted) line indicates the proton $h_{11 / 2}$ particle [550]1/2 trajectory.

and a neutron hole in the $g_{7 / 2}$ orbital. Accordingly, this isomer is associated with high- $K 8^{+}$state based on the $\pi g_{9 / 2}[404] 9 / 2 \nu g_{7 / 2}[404] 7 / 2$ configuration. Figure 19 summarizes the observed isomers in the low-lying level scheme of ${ }^{122} \mathrm{I}$.

Interestingly, an energetically favored $7^{+}$(or $8^{+}$) isomer with a half-life of $171 \mu \mathrm{s}$ has been observed at $596 \mathrm{keV}$ in ${ }^{126} \mathrm{Cs}$ [17]. Moreover, the ${ }^{124} \mathrm{Cs}$ nucleus also has the longlived $7^{+}$isomer with a half-life of $6.3 \mathrm{~s}$ at $463 \mathrm{keV}$ [17]. However, no level structures built on these two isomers have been identified. We propose that the $596-\mathrm{keV}$ isomer in ${ }^{126} \mathrm{Cs}$ may be $8^{+}$rather than $7^{+}$. Here, the $7^{+}$state can be built from either the $\pi g_{7 / 2} \nu g_{7 / 2}$ or $\pi g_{9 / 2} \nu g_{7 / 2}$ configurations. Taking the occurrence of the long-lived isomer into consideration, the $\pi g_{9 / 2}$ orbital should be involved in producing the $K=$ $8^{+}\left(\Omega_{p}+\Omega_{n}=9 / 2+7 / 2\right)$ state. In the $\beta$-decay scheme of ${ }^{126} \mathrm{Cs}$ from ${ }^{126} \mathrm{Ba}$, the $1^{+}$state, related to the Gamow-Teller

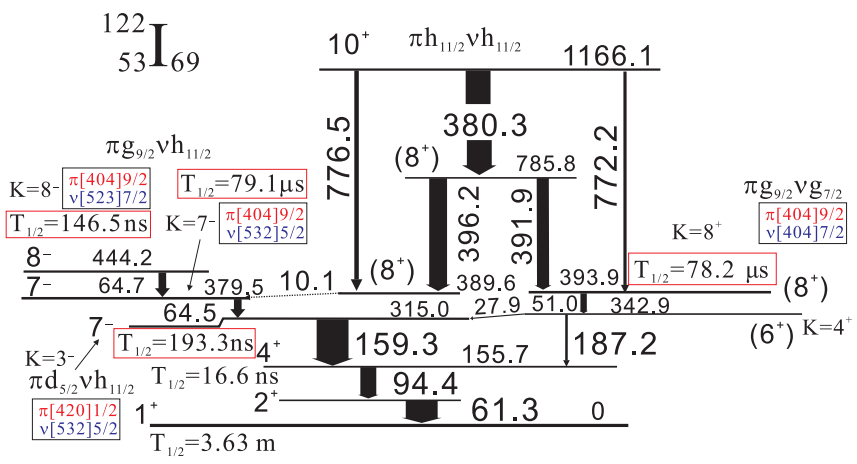

FIG. 19. The isomeric states identified in the present work for ${ }^{122} \mathrm{I}$. The corresponding proton-neutron shell configurations, Nilsson orbitals, and $K$-quantum numbers are indicated.

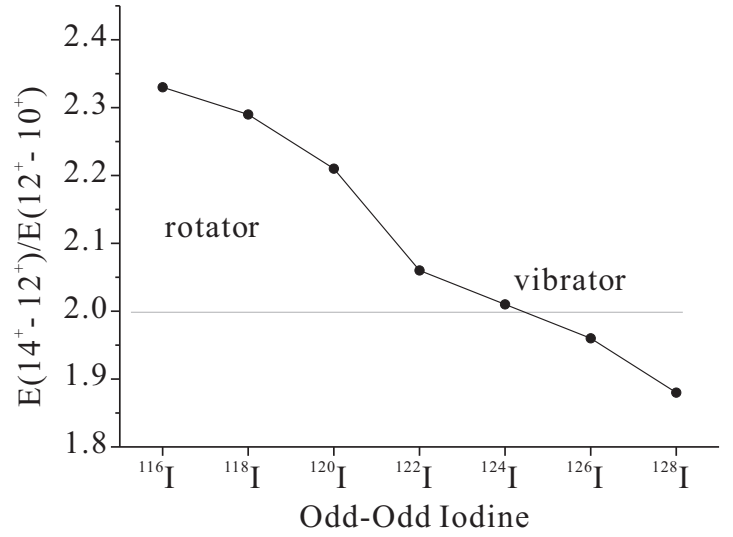

FIG. 20. Systematics of energy ratios of the $14^{+}$state to the $12^{+}$ state based on the $10^{+}$state built on the $\pi h_{11 / 2} v h_{11 / 2}$ configuration in ${ }^{116}$ I to ${ }^{128}$ I [9,20,21,27-29].

transition between proton-neutron spin doublet partners, has been found at $682 \mathrm{keV}$ [16], which lies close to the $595-\mathrm{keV}$ isomer in ${ }^{126} \mathrm{Cs}$. Further, in the $\beta$-decay scheme of ${ }^{122} \mathrm{Xe}$ to ${ }^{122} \mathrm{I}$, two $1^{+}$states at 350 and $417 \mathrm{keV}$, respectively, have also been observed [17]. These $1^{+}$states can be interpreted as being associated with the $\pi g_{9 / 2} \nu g_{7 / 2}$ configuration formed by the transformation of a proton in the $g_{9 / 2}$ orbital to a neutron in the $g_{7 / 2}$ orbital. Similarly, the isomer with a $6.3 \mathrm{~s}$ in ${ }^{124} \mathrm{Cs}$ is likely to be $8^{+}$instead of $7^{+}$. Then, all the isomers with $J^{\pi}=8^{+}$in ${ }^{122} \mathrm{I},{ }^{124} \mathrm{Cs}$, and ${ }^{126} \mathrm{Cs}$ can be interpreted in terms of the $K$ isomerism.

\section{B. Band 1}

Figure 20 shows the systematic trend for energy ratios of the $14^{+}$level to the $12^{+}$level in the $\pi h_{11 / 2} v h_{11 / 2}$ bands in ${ }^{116}$ I to ${ }^{128}$ I. This energy ratio corresponds to that of the first $4^{+}$to the first $2^{+}$states in even-even nuclei. This value, as a deformation parameter, evolves from $<2$ for a spherical nucleus through 2 for a vibrator to 3.3 for a deformed axial rotator. One can see that ${ }^{122} \mathrm{I}$ lies in a typical vibrator. According to total energy (Routhian) surface calculations, the energy surfaces based on the $\pi h_{11 / 2} \nu h_{11 / 2}$ configuration are very soft with respect to $\gamma$, showing a shallow minimum at a noncollective oblate shape with $\epsilon_{2}=0.16$ and $\gamma=60^{\circ}$. In this weak deformation, the $\pi h_{11 / 2}[550] 1 / 2 v h_{11 / 2}[523] 7 / 2$ and $\pi h_{11 / 2}[550] 1 / 2 \nu h_{11 / 2}[532] 5 / 2$ configurations correspond to the favored and unfavored states in band 1 .

Generally, the plot of energy $(E)$ versus angular momentum (total spin $J$ ) gives broad information on nuclear structures according to changes of line curves. If we take a derivative from such lines, we can get more visual patterns on the derived slopes. As shown in Fig. 21, the constant, monotonically increasing, irregular, and dip values, respectively, indicate vibrational, rotational, singles, and energetically favored multinucleon alignments modes. In Fig. 21, the following distinctive features are found: First, between $J^{\pi}=12^{+}$to $16^{+},{ }^{120} \mathrm{I}$ and ${ }^{122} \mathrm{I}$ indicate a rotator and a vibrator, respectively. Second, both ${ }^{120} \mathrm{I}$ and ${ }^{122} \mathrm{I}$ have irregular singles 


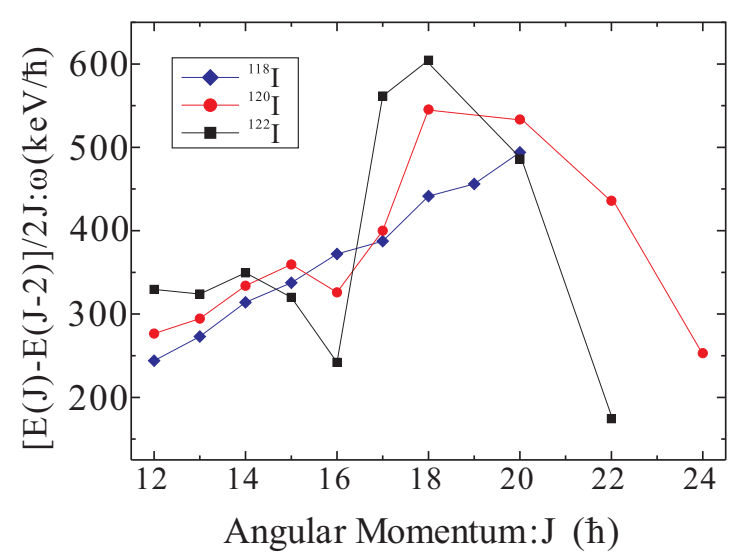

FIG. 21. Plots of angular frequency, $\omega(\mathrm{keV} / \hbar)$, vs angular momentum (spin $\hbar$ ) for the $\pi h_{11 / 2} v h_{11 / 2}$ bands of ${ }^{118} \mathrm{I}$ [20], ${ }^{120} \mathrm{I}$ [21], and ${ }^{122} \mathrm{I}$.

mode at $16^{+}$showing more dip values at $22^{+}$and $24^{+}$. In contrast, ${ }^{118}$ I keeps a rotational structure over a whole range.

In order to visualize the observed energetically favored states, in Fig. 22 we show the excitation energies in the form of a rigid rotor plot, from which a rotating liquid-drop energy reference equal to $\hbar^{2} / 2 \mathfrak{I}_{\text {rig }} J(J+1)$ has been subtracted. Here, the $\hbar^{2} / 2 \mathfrak{I}_{\text {rig }}$ is given by $35.8 A^{-5 / 3} \mathrm{MeV}$, which is $0.0119 \mathrm{MeV}$ for the ${ }^{122} \mathrm{I}$ case, and the $\mathfrak{I}_{\text {rig }}$ is the rigid body inertial momentum. From this plot, the $16^{+}, 22^{+}$, and $25^{+}$levels in band 1 as well as the $21^{-}$level in band 2 reveal the energetically favored states. The $16^{+}$state can be explained as being associated with two-proton alignment in the $g_{7 / 2}$ and $d_{5 / 2}$ orbitals coupled to the $10^{+}$state due to the $\pi h_{11 / 2} \nu h_{11 / 2}$ configuration such as the $\left[\pi\left(g_{7 / 2}\right)^{1}\left(d_{5 / 2}\right)^{1}\right]_{6^{+}}\left[\pi\left(h_{11 / 2}\right)^{1} v\left(h_{11 / 2}\right)^{1}\right]_{10^{+}}$configuration. As shown in Fig. 23, this four-nucleon fully alignment yields a noncollective oblate structure at $16^{+}$, having a deformation with $\beta_{2} \approx 0.18, \gamma \approx 60^{\circ}$. This configuration can be interpreted as being associated with three-proton and one-neutron alignments of the $\left[\pi\left(g_{7 / 2} d_{5 / 2} h_{11 / 2}\right)^{3}\right]_{23 / 2^{-}}\left[v\left(h_{11 / 2}\right)^{1}\right]_{9 / 2^{-}}$configuration. Another favored oblate state occurs at $15^{+}$with

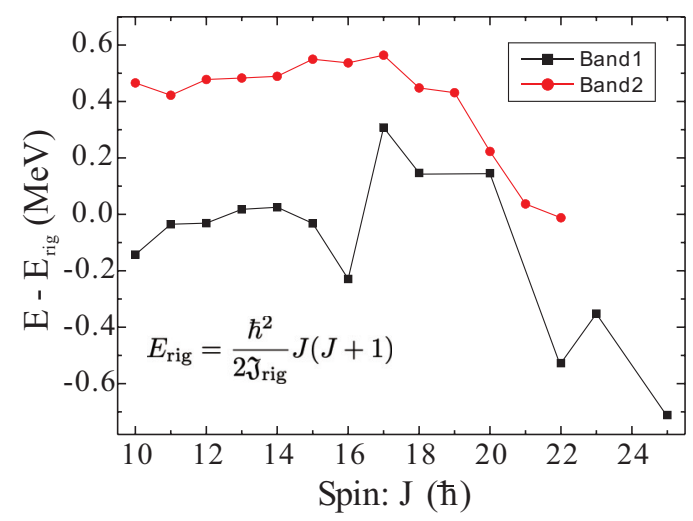

FIG. 22. Excitation energies in the form of a liquid-drop energy as a function of spin. Here, $E_{\text {rig }}$ is a rotating liquid-drop energy reference with $\hbar^{2} / 2 \mathfrak{I}_{\text {rig }}=0.0119 \mathrm{MeV}$ and $\mathfrak{I}_{\text {rig }}$ is the momentum of inertia.

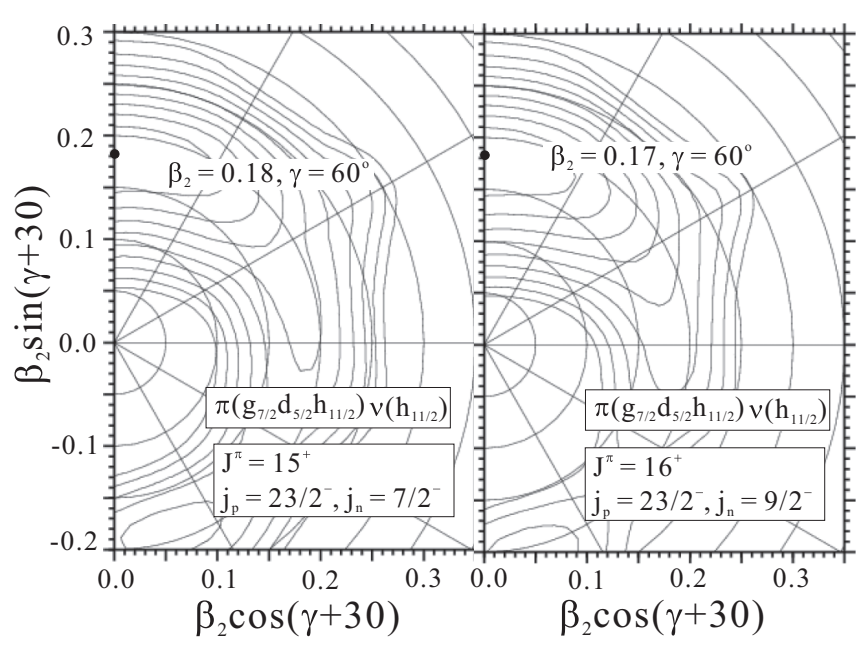

FIG. 23. Total Routhian surface (TRS) energy calculations for four quasiparticle configurations of $\pi\left(g_{7 / 2} d_{5 / 2} h_{11 / 2}\right)^{3} v\left(h_{11 / 2}\right)^{1}$ for ${ }^{122}$ I. Here, the energy minima on noncollective $\gamma=60^{\circ}$ axis occur at $15^{+}$and $16^{+}$which correspond to spin alignments caused by $\left[\pi\left(g_{7 / 2}\right)^{1}\left(d_{5 / 2}\right)^{1}\left(h_{11 / 2}\right)^{1}\right]_{23 / 2^{-}}\left[v\left(h_{11 / 2}\right)^{1}\right]_{7 / 2^{-}}$and $\left[\pi\left(g_{7 / 2}\right)^{1}\right.$ $\left.\left(d_{5 / 2}\right)^{1}\left(h_{11 / 2}\right)^{1}\right]_{23 / 2^{-}}\left[v\left(h_{11 / 2}\right)^{1}\right]_{9 / 2^{-}}$configurations, respectively.

the $\left[\pi\left(g_{7 / 2} d_{5 / 2} h_{11 / 2}\right)^{3}\right]_{23 / 2^{-}}\left[v\left(h_{11 / 2}\right)^{1}\right]_{7 / 2^{-}}$configuration. In turn, the energetically favored states are formed at $22^{+}$ and $25^{+}$by three-proton and three-neutron alignments, $\left[\pi\left(g_{7 / 2} d_{5 / 2} h_{11 / 2}\right)^{3}\right]\left[v\left(h_{11 / 2}\right)^{3}\right]$. The $25^{+}$state is due to the full alignment of three neutrons in the $h_{11 / 2}$ orbital, producing $j_{n}=27 / 2^{-}(11 / 2+9 / 2+7 / 2)$ while the $22^{+}$state is due to a partial alignment of $j_{n}=21 / 2^{-}(9 / 2+7 / 2+5 / 2)$. The total energy surfaces indicate a noncollective oblate shape at $25^{+}$with $\beta_{2} \approx 0.16, \gamma \approx 60^{\circ}$.

For understanding the levels above the $16^{+}$state in ${ }^{122} \mathrm{I}$, we make a further comparison with the spherical structure of ${ }^{118} \mathrm{Sn}$ and ${ }^{116} \mathrm{Sn}$ as shown in Fig. 24. The transition energies between the $20^{+}$and the $16^{+}$levels follow those of the ${ }^{118} \mathrm{Sn}$ core. Besides, the transitions of the $29^{+}$state to the $25^{+}$state are almost identical in energy to the ${ }^{116} \mathrm{Sn}$ core energies. Here, the $16^{+}$state is associated with the three-proton and one-neutron alignments outside the ${ }^{118}$ Sn core. Next, the $25^{+}$ state turns into a noncollective oblate structure owing to threeproton and three-neutron alignments outside the ${ }^{116} \mathrm{Sn}$ core. This comparison gives us insight into the emergence of similar transitional energies feeding the noncollective oblate states with those of the neighboring $\mathrm{Sn}$ cores. It is noteworthy to know that the $22^{+}$state also arises from three valence protons in the $g_{7 / 2}, d_{5 / 2}$, and $h_{11 / 2}$ orbitals and five valence neutrons in the $h_{11 / 2}$ orbital outside the ${ }^{114} \mathrm{Sn}$ core. However, among five neutrons in the $h_{11 / 2}$ orbital, a pair of two neutrons does not contribute to the aligned angular momentum.

If we turn our attention to an energetically favored state at $27^{-}$in the holmium nucleus ${ }^{154}$ Ho $(Z=67, N=87)$ [30], a similar pattern could be seen. This favored state is known to be formed by six valence nucleon alignments, $\pi\left(h_{11 / 2}\right)^{3} v\left(f_{7 / 2}\right)^{1}\left(h_{9 / 2}\right)^{1}\left(i_{13 / 2}\right)^{1}$, outside a semi-double-shell closure with $Z=64$ and $N=82,{ }^{146} \mathrm{Gd}$. Here, three protons in the $h_{11 / 2}$ orbital produce the maximal spins $J_{p}=27 / 2^{-}$ $(11 / 2+9 / 2+7 / 2)$ while three neutrons in the $f_{7 / 2}, h_{9 / 2}, i_{13 / 2}$ 


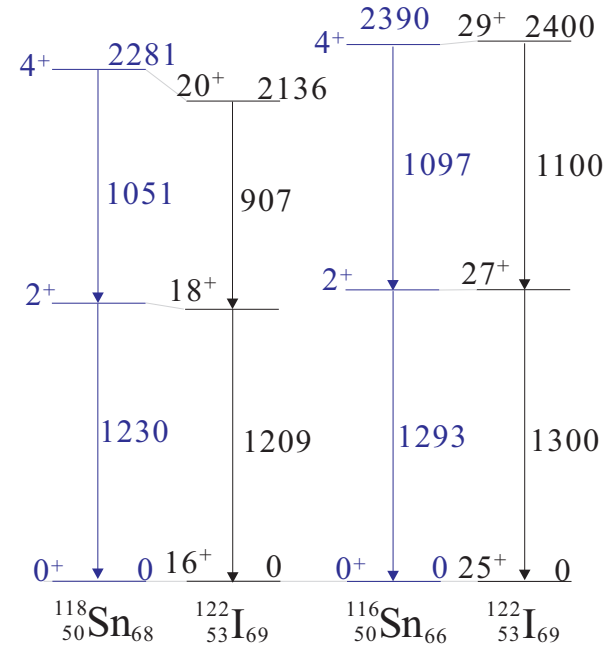

FIG. 24. Level comparisons between the ground band states of ${ }^{118} \mathrm{Sn}$ and the $20^{+}$to the $16^{+}$states of ${ }^{122} \mathrm{I}$ and the ground band states of ${ }^{116} \mathrm{Sn}$ and the $29^{+}$to $25^{+}$states of ${ }^{122} \mathrm{I}$. Notice that the referenced levels, $16^{+}$and $25^{+}$states, for ${ }^{122} \mathrm{I}$ are all set to be zero in energy. Energies are given in keV. The transitions of $29^{+}$to $27^{+}$in ${ }^{122} \mathrm{I}$ are quoted from Ref. [12].

space produce $J_{n}=27 / 2^{+}(7 / 2+9 / 2+11 / 2)$. Notice that among three neutrons in the $i_{13 / 2}$ orbital, two neutrons are under the pairing condition with $J=0$. In contrast, as discussed in Ref. [21], for ${ }^{152} \mathrm{Ho}$ three neutrons in the $f_{7 / 2}, h_{9 / 2}, i_{13 / 2}$ space yield a maximal angular momentum $29 / 2^{+}$, by which the most energetically favored state occurs at $28^{-}$, leading to a spin-trap isomeric state. For summarizing the emerging noncollective oblate states for both iodine and holmium, we show excitation energies of yrast states in the form of the rigid body reference in Fig. 25. One can see that the most energetically favored states are the $25^{+}$states in ${ }^{120} \mathrm{I}$ and ${ }^{122} \mathrm{I}$ and the $28^{-}$(or $27^{-}$) states in ${ }^{152} \mathrm{Ho}$ and ${ }^{154} \mathrm{Ho}$, respectively. Similar favored states also occurred at $16^{+}$in ${ }^{122} \mathrm{I}$ and ${ }^{120} \mathrm{I}$ and at $19^{-}$in ${ }^{154} \mathrm{Ho}$ and ${ }^{152} \mathrm{Ho}$, respectively.

\section{Band 2}

Band 2 represents a rotational-like feature built on the $10^{-}$ at $1775 \mathrm{keV}$. This high-lying bandhead energy, comparing to those of bands 3 to 6 , indicates that the structure should be related to the proton intruder $h_{11 / 2}$ orbital. Besides, this band has a coupled structure without a signature splitting. It means that a high- $K$ orbital should be involved in this collective state. As shown in Fig. 17, the trajectory of the neutron $g_{7 / 2}[404] 7 / 2$ orbital follows a deformed path like the proton $g_{9 / 2}[404] 9 / 2$ orbital. Accordingly, we suggest that band 2 should be associated with the configuration of a proton in an $h_{11 / 2}$ orbital coupled to the neutron $g_{7 / 2}$ orbital which is the $\pi h_{11 / 2} \nu g_{7 / 2}$ configuration. Our assignment is in agreement with Singh et al. [12]. We found that the cascade of transitions from $10^{-}$to $16^{-}(559-654-786 \mathrm{keV})$ in ${ }^{122} \mathrm{I}$ is similar to the cascade of $2^{+}$to $8^{+}(560-654-738 \mathrm{keV})$ in $\gamma$ band of ${ }^{122} \mathrm{Xe}$. Consequently, band 2 could be considered to be more related to the $\gamma$-based rotational structure than the ground deformed core structure.
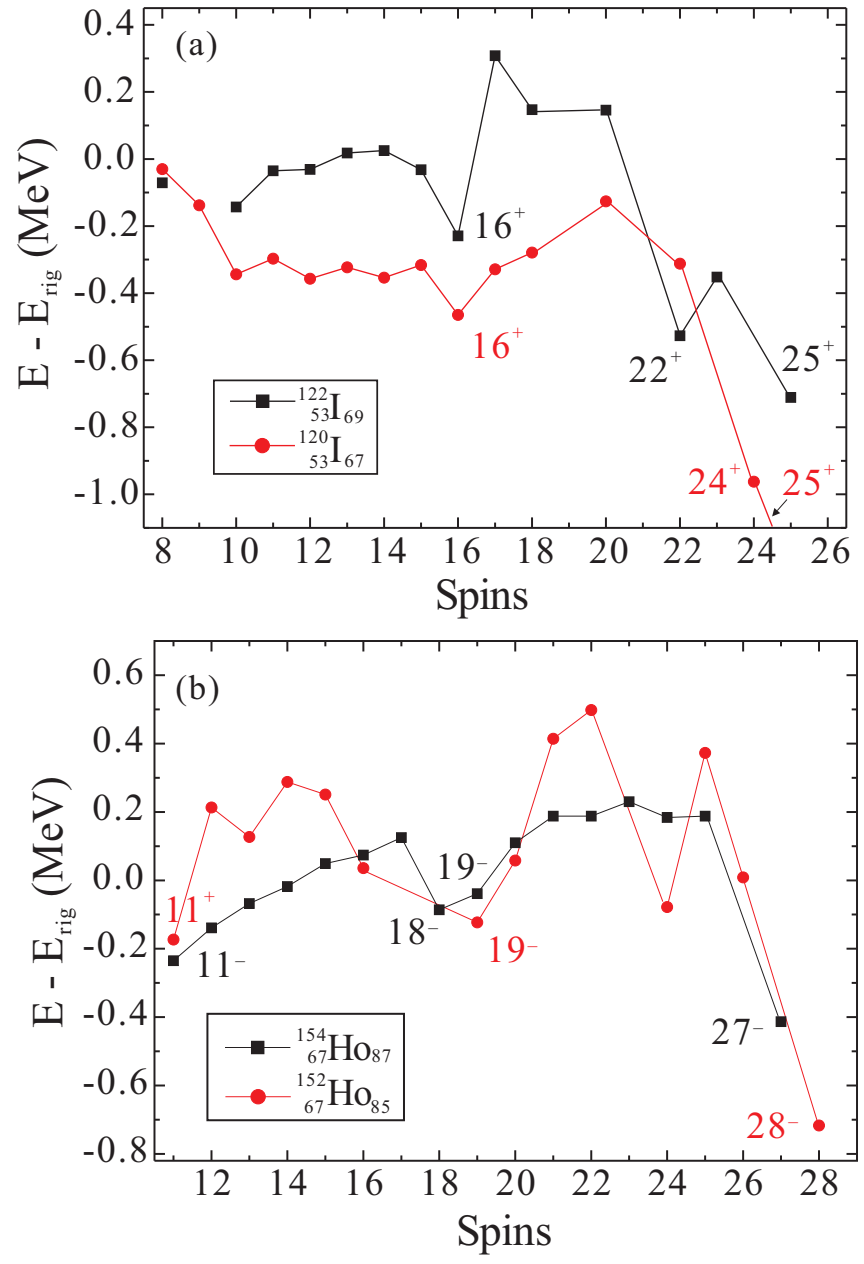

FIG. 25. Excitation energies of $E-E_{\text {rig }}$ in the form of a liquiddrop energy as a function of spin for (a) ${ }^{122} \mathrm{I}$ and ${ }^{120} \mathrm{I}$ and (b) ${ }^{154} \mathrm{Ho}$ and ${ }^{152}$ Ho. Here, $E_{\text {rig }}$ is a rotating liquid-drop energy reference with $\hbar^{2} / 2 \mathfrak{I}_{\text {rig }}=35.8 A^{-5 / 3} \mathrm{MeV}$ and $\mathfrak{I}_{\text {rig }}$ is the momentum of inertia.

We notice a small difference between the transitional energies of the $27 / 2^{-}$to $23 / 2^{-}$in ${ }^{121}$ I and the $16^{-}$to $14^{-}$in ${ }^{122} \mathrm{I}$, implying a structural change at $14^{-}$. It was found that the transitions feeding $14^{-}$are more intense in population than the below transitions. Further, by observing a coupled structure up to $17^{-}$, the states above $16^{-}$would be different from the below states. Finally, the $20^{-}, 21^{-}$, and $22^{-}$states show an irregular feature, indicate a noncollective structure. The $14^{-}$level is most likely due to the four quasiparticle alignment based on the $\pi\left(h_{11 / 2}\right) v\left(g_{7 / 2}\right)$ orbital coupled by two-proton alignment in the $g_{7 / 2}$ and $d_{5 / 2}$ orbitals, the $\left[\pi\left(h_{11 / 2}\right) v\left(g_{7 / 2}\right)\right]_{8^{-}}\left[\pi g_{7 / 2} d_{5 / 2}\right]_{6^{+}}$configuration. In turn, the irregular states are formed in singles mode by aligning six nucleonic orbitals of three protons and three neutrons in the $\pi\left[\left(g_{7 / 2} d_{5 / 2} h_{11 / 2}\right)\right]_{23 / 2^{-}} v\left[d_{3 / 2}\left(h_{11 / 2}\right)^{2}\right]_{19 / 2^{+}}$configuration. The weakness of coupled structure above $16^{-}$implies that a neutron in the $g_{7 / 2}$ orbital should be replaced by a neutron in a $d_{3 / 2}$ orbital. As shown in Figs. 17 and 18, at small deformations around $\epsilon_{2}=0.16$ and $\gamma=0^{\circ}$, the specific Nilsson configurations in the neutron $h_{11 / 2}, d_{3 / 2}, s_{1 / 2}$, and $g_{7 / 2}$ orbitals are lying together at the lowest energy level. However, 

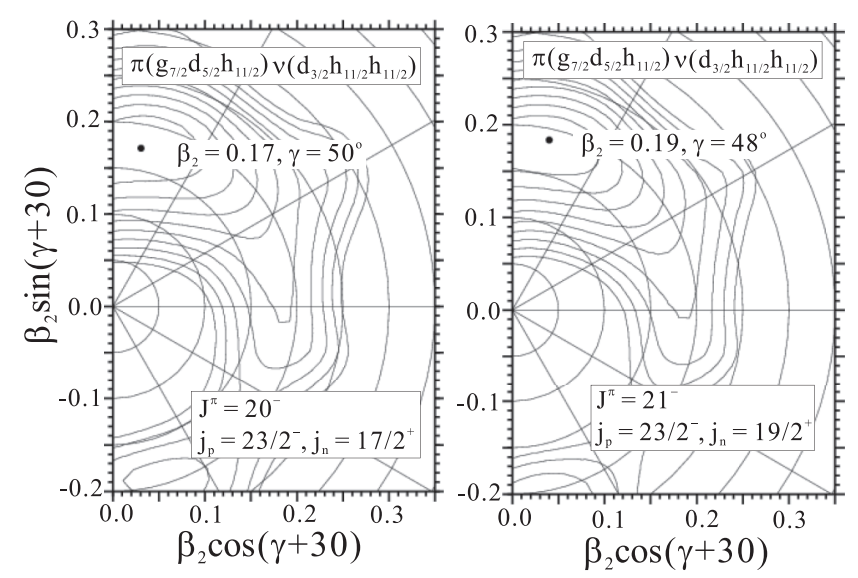

FIG. 26. Plots of calculated total energy surfaces for ${ }^{122} \mathrm{I}$, at $J^{\pi}=20^{-}$and $21^{-}$by six quasiparticle alignments based on the $\pi\left[\left(g_{7 / 2}\right)^{1}\left(d_{5 / 2}\right)^{1}\left(h_{11 / 2}\right)^{1}\right] \nu\left[\left(d_{3 / 2}\right)^{1}\left(h_{11 / 2}\right)^{2}\right]$ configuration, when an angular frequency with $0.18 \mathrm{MeV} / \hbar$.

as $\gamma$ values increase toward $60^{\circ}$, the $g_{7 / 2}[404] 7 / 2$ orbital deviates from such a position. In contrast, for protons, the $d_{5 / 2}[420] 1 / 2, g_{7 / 2}[422] 3 / 2$, and $h_{11 / 2}[550] 1 / 2$ orbitals keep their energetically favored character.

Figure 26 shows the total energy (Routhian) surface calculations for three protons and three neutrons, leading to the $\pi\left(g_{7 / 2} d_{5 / 2} h_{11 / 2}\right)^{3} v\left(d_{3 / 2}\right)^{1}\left(h_{11 / 2}\right)^{2}$ configuration. This configuration favors a near oblate shape with $\beta_{2} \approx 0.17$ and $\gamma \approx 50^{\circ}$ as indicated by an energy minima at $J^{\pi}=20^{-}$and $21^{-}$. These $\gamma$-soft minima close to the noncollective oblate shape of $\gamma=$ $60^{\circ}$ are reasonable for explaining the observed energetically favored $21^{-}$state and occurrence of an irregular structure between $20^{-}$and $22^{-}$. Consequently, the most favored $21^{-}$ state comes from the spin alignments of $j_{p}=23 / 2^{-}(7 / 2+$ $5 / 2+11 / 2)$ and $j_{n}=19 / 2^{-}(3 / 2+9 / 2+7 / 2)$.

Interestingly, a band-2-like structure also has been observed in the ${ }^{124} \mathrm{Cs}$ isotone $[31,32]$. Figure 27 plots the angular frequency versus angular momentum curves for two bands. It is found that up to $17^{-}$they show a similar pattern except at

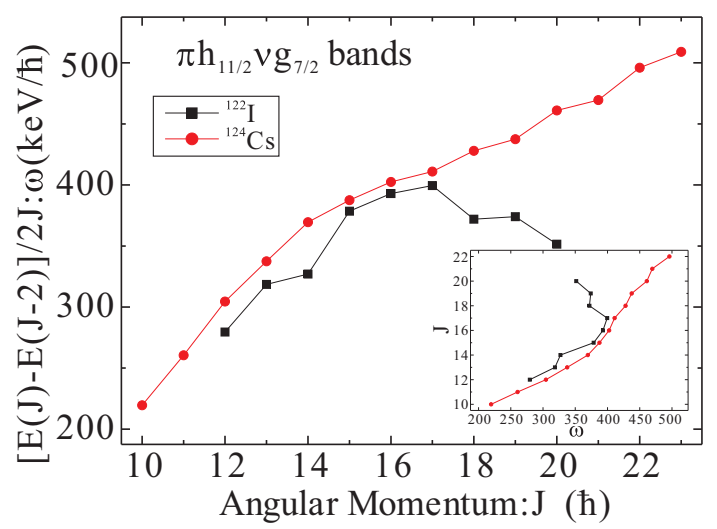

FIG. 27. Comparison of curves of angular frequency, $\omega(\mathrm{keV} / \hbar)$, as a function of angular momentum (spin $\hbar$ ) for band 2 associated with the $\pi h_{11 / 2} v g_{7 / 2}$ in ${ }^{122} \mathrm{I}$ and ${ }^{124} \mathrm{Cs}$ [31]. Inset represents angular momentum with respect to angular frequency.
$14^{-}$. But the states above $17^{-}$are apparently different: One, ${ }^{122} \mathrm{I}$, goes down to singles mode and the other, ${ }^{124} \mathrm{Cs}$, continues a deformed mode. For the case of ${ }^{124} \mathrm{Cs}$, we notice that the curve splits in slope at $14^{-}$. As shown in Fig. 27, a gain in alignment is found to be six units, from $8^{-}$to $14^{-}$. This comes from the two-proton alignment in the $g_{7 / 2}$ and $d_{5 / 2}$ orbitals. Next, up to $22^{-}$, the two-neutron alignment in the $h_{11 / 2}$ orbital follows. There is no occurrence of band-2-like structure above and below $N=69$ in I and Cs, and it is interesting that only at $N=69$ will a neutron hole in the $g_{7 / 2}$ orbital and a proton particle in the $h_{11 / 2}$ orbital give rise to a coupled rotational band.

\section{Bands 3 to 9}

Bands 3 to 8 represent a deformed rotational structure. We suggest that these bands are due to the $\pi d_{5 / 2} v h_{11 / 2}$ and $\pi g_{7 / 2} \nu h_{11 / 2}$ configurations. Bands 3 and 4 form signature partners, indicating a weak rotational structure built on the $8^{-}$and $9^{-}$, respectively. As already pointed out, at small deformations, the deformed shell orbitals of $g_{7 / 2}[422] 3 / 2$ and $d_{5 / 2}[420] 1 / 2$ are almost degenerate in the ground level for protons. In contrast, for the $h_{11 / 2}$ neutrons, the [532]5/2 and $[523] 7 / 2$ orbitals are close to the neutron ground level. Thus, bands 3 and 4 are considered to be related to the $\pi[422] 3 / 2 v[532] 5 / 2$ (or [523]7/2) configuration. Then, bands 5 and 6 are associated with the $\pi[420] 1 / 2 \nu[532] 5 / 2$ (or [523]7/2) configuration. Our assignments are consistent with the results given by Singh et al. [12].

Band 7 has been known to be built on a proton hole $g_{9 / 2}$ coupled to a neutron $h_{11 / 2}$, the $\pi g_{9 / 2} \nu h_{11 / 2}$ configuration. In the region of nuclei around the $Z=50$ shell gap, both the $g_{9 / 2}$ and $h_{11 / 2}$ intruder orbitals are close to Fermi surfaces. As was discussed earlier, the $8^{-}$state at $444 \mathrm{keV}$ is related to the $\pi g_{9 / 2}[404] 9 / 2 v h_{11 / 2}[523] 7 / 2$ configuration, leading to the $K^{\pi}=8^{-}$isomer. According to the present deformed shell-model calculations as shown in Fig. 17, band 7 is more deformed near $\epsilon_{2}=0.24$ than other collective bands. Band 8 indicates a partner band of band 7. The energy differences between bands 7 and 8 are found to be around $200 \mathrm{keV}$. In odd-mass I including ${ }^{121} \mathrm{I}$, the second negative-parity bands based on the $\pi g_{9 / 2}$ configuration were observed and interpreted as being attributed to the coupling with $\gamma$ vibration. The observation of such side bands built on a $\gamma$ vibration may be explained in terms of the coupling of the $\pi g_{9 / 2} \nu h_{11 / 2}$ configuration to the $\gamma$ phonon.

Band 9 can be considered to be a collective structure based on the $\pi\left(g_{7 / 2}\right.$ or $\left.d_{5 / 2}\right) v d_{3 / 2}$ configuration. This collective band develops as neutron number increases such as at ${ }^{124} \mathrm{I}$ [9] and at ${ }^{126} \mathrm{I}[9]$.

\section{CONCLUSION}

In this paper, we have studied the excited levels for odd-odd ${ }^{122} \mathrm{I}$. The excited states of ${ }^{122} \mathrm{I}$ were produced by the fusion-evaporation ${ }^{120} \mathrm{Sn}\left({ }^{7} \mathrm{Li}, 5 \mathrm{n}\right){ }^{122} \mathrm{I}$ reaction at a beam energy of $58 \mathrm{MeV}$. By using both in-beam and out-of-beam $\gamma$-ray spectroscopic techniques, we have successfully established excitation energies for the observed levels. Numerous isomeric states have been identified with half-lives of a few 
tens of ns to a few tens of $\mu$ s. Among them, two isomers with identical half-lives around $80 \mu \mathrm{s}$ can be interpreted in terms of $K$ isomerism; one is due to $K^{\pi}=7^{-}$based on the $\pi g_{7 / 2}[404] 9 / 2 v h_{11 / 2}[532] 5 / 2$ configuration and the other is due to $K^{\pi}=8^{+}$based on the $\pi g_{9 / 2}[404] 9 / 2 \nu g_{7 / 2}[404] 7 / 2$ configuration. We showed that the level structure of the low-lying spin states can be described in terms of the spherical shell model with the configurations of protons in the full space and five or seven valence neutrons constrained in the $h_{11 / 2}, d_{3 / 2}$, and $s_{1 / 2}$ orbitals. The positive-parity collective band built on the $\pi h_{11 / 2} v h_{11 / 2}$ configuration represents a quadrupole vibrational structure. In this band, a noncollective oblate structure was observed at spin parities of $16^{+}, 22^{+}$, and $25^{+}$, respectively, which are associated with multinucleon alignments of the $\left[\pi\left(g_{7 / 2}\right)^{1}\left(d_{5 / 2}\right)^{1}\right]_{6^{+}}\left[\pi\left(h_{11 / 2}\right)^{1} v\left(h_{11 / 2}\right)^{1}\right]_{10^{+}}$, $\pi\left[\left(g_{7 / 2}\right)^{1}\left(d_{5 / 2}\right)^{1}\left(h_{11 / 2}\right)^{1}\right]_{23 / 2^{-}} v\left[\left(h_{11 / 2}\right)^{3}\right]_{21 / 2^{-}}$, and $\pi\left[\left(g_{7 / 2}\right)^{1}\right.$ $\left.\left(d_{5 / 2}\right)^{1}\left(h_{11 / 2}\right)^{1}\right]_{23 / 2^{-}} v\left[\left(h_{11 / 2}\right)^{3}\right]_{27 / 2^{-}}$configurations. In contrast, the collective states based on the $\pi h_{11 / 2} \nu g_{7 / 2}$ configuration showed a triaxial deformed structure. This is a unique case of both a collective vibration and a collective rotation occurring built on the proton $h_{11 / 2}$ orbital in the odd-odd I isotopes.

\section{ACKNOWLEDGMENTS}

We would like to thank R. Wood for his help. This work was supported by the Rare Isotope Science Project (RISP) of the Institute for Basic Science (IBS) funded by the Ministry of Science, ICT and Future Planning (MSIP) and by the National Research Foundation (NRF) of the Republic of Korea (Grant No. 2013M7A1A1075764). This work was also supported by the National Research Foundation (NRF) of the Republic of Korea grant funded by the Ministry of Science, ICT and Future Planning (MSIP) (Grant No. 2018R1A5A1025563) and the National Natural Science Foundation of China (Grant No. 11775316).
[1] C.-B. Moon, T. Komatsubara, and K. Furuno, J. Korean Phys. Soc. 43, 319 (2003).

[2] C.-B. Moon, T. Komatsubara, T. Shizuma, Y. Sasaki, H. Ishiyama, T. Jumatsu, and K. Furuno, Eur. Phys. J. A 5, 13 (1999).

[3] S. Törmanäen, S. Juutinen, R. Julin, A. Lampinen, E. Mäkelä, M. Piiparinen, A. Savelius, A. Virtanen, G. B. Hagemann, C. Droste et al., Nucl. Phys. A 613, 282 (1997).

[4] Y. Liang, D. B. Fossan, J. R. Hughes, D. R. LaFosse, T. Lauritsen, R. Ma, E. S. Paul, P. Vaska, M. P. Waring, and N. Xu, Phys. Rev. C 45, 1041 (1992).

[5] L. G. Kostova, W. Andrejtscheff, L. K. Kostov, F. Dönau, L. Käubler, H. Prade, and H. Rotter, Nucl. Phys. A 485, 31 (1988).

[6] H. Sharma, B. Sethi, R. Goswami, P. Banerjee, R. K. Bhandari, and J. Singh, Phys. Rev. C 59, 2446 (1999).

[7] R. E. Shroy, D. M. Gordon, M. Gai, D. B. Fossan, and A. K. Gaigalas, Phys. Rev. C 26, 1089 (1982).

[8] B. Ding, Y. H. Zhang, X. H. Zhou, G. X. Dong, F. R. Xu, M. L. Liu, G. S. Li, N. T. Zhang, H. X. Wang, H. B. Zhou et al., Phys. Rev. C 85, 044306 (2012).

[9] C.-B. Moon, G. D. Dracoulis, R. A. Bark, A. P. Byrne, P. A. Davidson, A. N. Wilson, T. Kibédi, and G. J. Lane, Department of Nuclear Physics Annual Report, ANU-P/1564, Australian National University, 2002, pp. 11-17.

[10] C.-B. Moon, G. D. Dracoulis, R. A. Bark, A. P. Byrne, P. A. Davidson, A. N. Wilson, A. M. Baxter, T. Kibédi, and G. J. Lane, J. Korean Phys. Soc. 43, S100 (2003).

[11] C.-B. Moon, G. D. Dracoulis, R. A. Bark, A. P. Byrne, P. A. Davidson, T. Kibédi, G. J. Lane, and A. N. Wilson, J. Korean Phys. Soc. 59, 1525 (2011).

[12] P. Singh, S. Nag, K. Selvakumar, A. K. Singh, I. Ragnarsson, A. Bisoi, A. Goswami, S. Bhattacharya, S. Kumar, K. Singh et al., Phys. Rev. C 85, 054311 (2012).

[13] G.-Y. Liu, L. Li, X.-F. Li, D.-Y. Yu, J. Sun, C.-B. Li, Y.-J. Ma, X-G. Wu, C.-Y. He, Y. Zheng, L.-H. Zhu, and Y.-X. Zhao, Chin. Phys. Lett. 29, 092301 (2012).

[14] H. Kaur, J. Singh, A. Sharma, D. Mehta, N. Singh, P. N. Trehan, H. C. Jain, S. D. Paul, E. S. Paul, and R. K. Bhowmik, Phys. Rev. C 55, 2234 (1997).

[15] K. Kitao, M. Kitao, Z. Matsumoto, and T. Seo, Nucl. Data Sheets 49, 315 (1986).
[16] C. Y. Wu, M. Satteson, K. Wang, D. Cline, H. E. Gove, S. S. Hanna, M. Hass, R. Ibbotson, and A. V. Ramayya, Phys. Rev. C 41, 1600 (1990).

[17] National Nuclear Data Center, Brookhaven National Laboratory, http://www.nndc.bnl.gov/.

[18] B. A. Brown, N. J. Stone, J. R. Stone, I. S. Towner, and M. Hjorth-Jensen, Phys. Rev. C 71, 044317 (2005).

[19] N. Shimizu, arXiv:1310.5431.

[20] C.-B. Moon, T. Komatsubara, T. Shizuma, Y. Sasaki, K. Furuno, and C. S. Lee, Nucl. Phys. A 728, 350 (2003).

[21] B. Moon, C.-B. Moon, G. D. Dracoulis, R. A. Bark, A. P. Byrne, P. A. Davidson, G. J. Lane, T. Kibédi, A. N. Wilson, C. Yuan, and B. Hong, Phys. Lett. B 782, 602 (2018).

[22] S. G. Nilsson, C. F. Tsang, A. Sobiczewski, Z. Szymański, S. Wycech, C. Gustafson, I.-L. Lamm, P. Möller, and B. Nilsson, Nucl. Phys. A 131, 1 (1969).

[23] S. G. Nilsson and I. Ragnarsson, Shapes and Shells in $\mathrm{Nu}$ clear Structure (Cambridge University Press, Cambridge, UK, 1995).

[24] M. Wang, G. Audi, A. H. Wapstra, F. G. Kondev, M. MacCormik, X. Xu, and B. Pfeiffer, Chin. Phys. C 36, 1603 (2012).

[25] R. Wyss, J. Nyberg, A. Johnson, R. Bengtsson, and W. Nazarewicz, Phys. Lett. B 215, 211 (1988).

[26] W. Nazarewicz, R. Wyss, and A. Johnson, Nucl. Phys. A 503, 285 (1989).

[27] B. Kanagalekar, P. Das, B. Bhujang, S. Muralithar, R. P. Singh, and R. K. Bhowmik, Phys. Rev. C 88, 054306 (2013).

[28] H.-X. Wang, Y.-H. Zhang, B. Ding, X.-H. Zhou, N-T. Zhang, M.-L. Liu, Y.-J. Ma, Y. Sasakiz, K. Yamada, H. Ohshima et al., Chin. Phys. Lett. 27, 082701 (2010).

[29] C.-B. Moon, J. Korean Phys. Soc. 44, 244 (2004).

[30] C.-B. Moon, T. Komatsubara, and K. Furuno, Phys. Rev. C 88, 044308 (2013).

[31] K. Selvakumar, A. K. Singh, C. Ghosh, P. Singh, A. Goswami, R. Raut, A. Mukherjee, U. Datta, P. Datta, S. Roy, G. Gangopadhyay et al., Phys. Rev. C 92, 064307 (2015).

[32] A. K. Singh, A. Basu, S. Nag, H. Hübel, J. Domscheit, I. Ragnarsson, A. Al-Khatib, G. B. Hagemann, B. Herskind, D. R. Elema et al., Phys. Rev. C 97, 024323 (2018). 